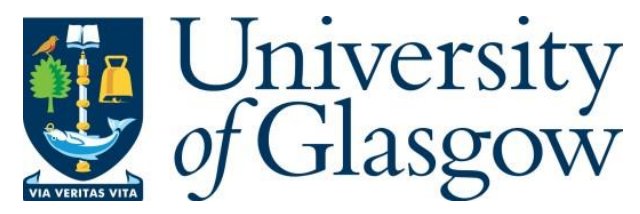

Ukai, T., Ohtani, K., and Obayashi, S. (2016) Turbulent jet interaction with a long risetime pressure signature. Applied Acoustics, 114, pp. 179-190.

(doi:10.1016/j.apacoust.2016.07.015)

There may be differences between this version and the published version. You are advised to consult the publisher's version if you wish to cite from it.

http://eprints.gla.ac.uk/129716/

Deposited on: 10 October 2016

Enlighten - Research publications by members of the University of Glasgow http://eprints.gla.ac.uk 


\title{
Turbulent Jet Interaction with a Long Rise-Time Pressure Signature
}

\author{
Takahiro Ukai ${ }^{1,2^{*}}$, Kiyonobu Ohtani ${ }^{1}$, Shigeru Obayashi ${ }^{1}$ \\ ${ }^{1}$ Institute of Fluid Science, Tohoku University, 2-1-1 Katahira, Aoba-ku, Sendai, Miyagi, 980- \\ 8577, Japan \\ ${ }^{2}$ School of Engineering, University of Glasgow, Glasgow, Scotland G12 8QQ, UK \\ ${ }^{*}$ Corresponding author: Takahiro Ukai \\ email: Takahiro.Ukai@glasgow.ac.uk
}

Telephone and Fax: + 44 - (0)141 - $330-2477$

\begin{abstract}
:
A sonic boom signature with a long rise time has the ability to reduce the sonic boom, but it does not necessarily minimize the sonic boom at the ground level because of the real atmospheric turbulence. In this study, an effect of the turbulence on a long rise-time pressure signature was experimentally investigated in a ballistic range facility. To compare the effects of the turbulence on the long and short rise-time pressure signatures, a cone-cylinder projectile that simultaneously produces these pressure signatures was designed. The pressure waves interacted with a turbulent field generated by a circular nozzle. The turbulence effects were evaluated using flow diagnostic techniques: high-speed schlieren photography, a point-diffraction interferometer, and a pressure measurement. In spite of the fact that the long and short rise-time pressure signatures simultaneously travel through the turbulent field, the turbulence effects do not give the same contribution to these overpressures. Regarding the long risetime pressure signature, the overpressure fluctuation due to the turbulence interaction is almost uniform, and a standard deviation 1.5 times greater than that of the no-turbulence case is observed. By contrast, a short rise-time pressure signature which passed through the same turbulent field is strongly affected by the turbulence. A standard deviation increases by a factor of 14 because of the turbulence interaction. Additionally, there is a non-correlation between the overpressure fluctuations of the long and short rise-time pressure signatures. These results deduce that the length of the rise time is important to the turbulence effects such as the shock focusing/diffracting.
\end{abstract}

\section{Keywords:}

Shock-turbulence interaction, Low sonic boom signature, Ballistic range facility 


\section{Introduction}

Regarding sonic boom phenomena, the effects due to the real atmosphere are an unavoidable issue. A typical sonic boom is produced by the shock waves generated from a supersonic transport vehicle traveling faster than sound. The shock waves pass through the real atmosphere and reach ground level, producing a large noise. The development of a supersonic transport vehicle with a low sonic boom level is necessary for reducing this noise $[1,2]$; however, this is not easy to achieve. Sonic boom signatures are affected by atmospheric conditions such as the turbulence, humidity, and temperature [3-6]. These atmospheric conditions cannot be controlled, and thus, they must be considered for the development of a robust, low sonic boom supersonic transport vehicle. The atmospheric effects must be well understood because these conditions are extremely important for accurately estimating the sonic boom magnitude.

The turbulence inside the atmospheric boundary layer has a significant effect on a normal N-shaped sonic boom signature. After the shock wave passes the real atmospheric turbulent field, the pressure waveforms dramatically change in shape, appearing as a spike, round, or multi-peak [7, 8]. According to flight-test results, the overpressure and the rise time, which are generally defined as sonic boom characteristics, vary randomly because of the turbulence, and a distorted pressure waveform with the double magnitude of the peak pressure level appears infrequently [9]. Additionally, the strong atmospheric turbulence leads to a high probability of these deformed waveforms; the deformation depends on the turbulence intensity. According to a laboratory-scale experiment, in the case of a shockturbulence interaction, the mean overpressure decreases, the mean rise time increases, and the standard deviations increase [10]. The larger standard deviations suggest that the large overpressure and short rise time may occur. Additionally, the turbulence effects are enhanced if a shock wave propagates through a turbulent field over a long distance [11]. The effects of the turbulence on a normal N-shaped sonic boom have been investigated [12-18]; however, they are not yet fully understood. Even though their behaviors have been elucidated, the turbulence effects on low sonic boom signatures are incomprehensible because these are special pressure signatures such as a flat-top overpressure and a long rise time.

The low sonic boom signature is achieved by the modification of a near-field pressure waveform, which is useful for sonic boom reduction [19]. A geometric fuselage design determines the shape of the near-field pressure waveform. McLean and Shrout [20] demonstrated the effectiveness of the nearfield pressure-signature modification technique for sonic boom minimization theoretically and by a wind tunnel test. The suitable near-field pressure distribution can be achieved by the modification of an airplane configuration for the sonic boom minimization, and this distribution persists through 
propagation to the far-field [21]. A blunt-nose configuration yields the minimum far-field overpressure and the minimum impulse at all distances; however, a high drag occurs because of the large shock strength in front of the nose. On the other hand, a flat-shaped sonic boom signature is produced by the adjustment of the airplane configuration and leads to the sonic boom minimization at the near- and mid-fields [20, 22, 23]. However, at a strict far-field, this signature has a higher overpressure magnitude than that of a blunt nose. This modification, which yields the flat-shaped signature, does not necessarily result in a higher drag occurrence. Additionally, it does not require a sufficient length with a slender configuration to obtain the near-field effect. A longer airplane with a slender configuration which is the other design concept yields a finite rise-time signature [20,22]. This is the most advantageous for the sonic boom minimization because the rise time is an important factor for the human response; a long rise time may eliminate the associated noise [22]. Additionally, the extremely long, slender configuration permits extending the near-field signature to the far-field.

These low sonic boom signatures may not be sufficiently maintained at ground level because of the turbulence although the airplane-design modification technique is effective for sonic boom reduction. This is because the real atmospheric conditions are not considered in the theory used for the modification technique. To prove the sonic boom reduction using the theory, a supersonic vehicle to which the modification technique was applied for generating a flat-top pressure signature performed a flight in the real atmosphere [24]. The flat-top shaped waveform was compared with the N-shaped wave under similar turbulence conditions to evaluate the effectiveness of the theory. Flight-test results [25] indicated that the flat-top waveform persisted in spite of a distorted waveform due to the turbulence interaction; thus, the sonic boom reduction due to the geometric modification was demonstrated. However, the distortion of the flat-top shape was clearly observed [26]. The turbulence effects of the flat-top signature resembled that of the normal $\mathrm{N}$-shaped signature. Although its behavior is not understood yet, the shock wave front might be distorted by the turbulence interaction even though the low sonic boom signature, in the case of the short rise-time pressure signature; i.e., the length of a rise time might relate to the distortion of a shock wave front. This is because the flat-top signature has the short front shock rise time that is observed in the normal $\mathrm{N}$-shaped signature. Although this problem is not directly addressed to elucidate this mechanism, many studies on the shock-turbulence interaction have been conducted for a short rise-time pressure signature.

Knowledge obtained from these research findings does not necessarily fully contribute the effect of the turbulence on long rise-time pressure signatures. In the general turbulence interaction with the short rise-time pressure signature, the shock wave focusing/diffracting at a shock wave front are caused by the shock wave propagating to various direction resulting from the distortion of the shock wave 
front [27, 28]. Salze et al. [29] showed a shock propagation distance relates to a transverse shock focusing region and experimentally estimated its shock focal region. Averiyanov et al. $[28,30]$ showed that an overpressure decrease and the arrival time are governed by the large scale of a velocity fluctuation, and the small scales of a velocity fluctuation mainly cause increasing the rise time. In the typical model experiments $[10,29,30]$, in order to simulate the $\mathrm{N}$-shaped sonic boom propagation in the real atmosphere, the characteristic length scales, such as a wavelength, a turbulent interaction distance, and the geometrical turbulence length scales, were adjusted. A generated N-shaped pressure waveform in the model experiments is categorized as a short rise time signature. Its front shock rise time which is unaffected by the turbulence interaction are less than approximately $3 \mu \mathrm{s}$, and it corresponds with a wave duration on the order of less than 1/10; thus, the short shock rise time is in high frequency domain. Wave attenuation, which is important factor for a shock wave propagation, depends on the frequency component which the pressure waveform has [31]. The frequency component of the short rise time does not correspond with that of the long rise time enable us to deduce that the same shock wave focusing/diffracting effects do not necessarily contribute to the long risetime pressure signature. To elucidate the effects of the length of the rise time on the shock-turbulence interaction phenomena, laboratory-scale experiments are effective because the turbulent parameters, such as the turbulence intensity and length scales, can be easily adjusted.

Ballistic range facilities have the ability to simulate the shock-turbulence interaction regarding the long rise-time pressure signature. To investigate the effect of the turbulence on a long rise-time pressure signature, the special pressure signatures must be generated in stationary ambient air. Ballistic range facilities that launch various geometric models can produce a special pressure signature because the near-field pressure waveforms change according to the model geometry; i.e., it is possible to launch a projectile with the near-field pressure waveform modified as a long rise-time signature. Additionally, the turbulent parameters can be controlled in the ballistic range facility because projectiles fly within stationary ambient air. On the other hand, supersonic wind tunnels are inadequate, as it is difficult to control the turbulent parameters because of the supersonic flow within a test section. Moreover, the shock generators, energy deposition by a spark or laser and shock tubes, are not useful for investigating the effects of the turbulence on a special pressure signature because they cannot modify the pressure waveforms. In a previous study, Hall [32] conducted a ballistic range experiment to investigate the effect of the turbulence on an N-shaped wave. The pressure characteristics affected by the turbulence resembled the flight-test results; however, the impinged pressure signature was $\mathrm{N}$-shaped because a bullet-type projectile was used.

A sonic boom signature with a long rise time has the ability to reduce the sonic boom level, but it 
does not necessarily minimize the sonic boom at the ground level because of the real atmospheric turbulence. It is important to evaluate the robustness of a long rise-time pressure signature, and its behavior propagating trough the turbulence must be investigated. In this study, the turbulence effects on a long rise-time pressure signature were experimentally investigated in a ballistic range facility. The long and short rise-time pressure signatures passed through the same turbulent field, and we compared these turbulence effects. The long and short rise-time pressure signatures were simultaneously produced by launching a cone-cylinder projectile. To evaluate the experimental setup, the turbulence effects on an $\mathrm{N}$-shaped wave were investigated using a cylindrical projectile. The flow diagnostics: high-speed schlieren photography, a point-diffraction interferometer (PDI), and a pressure measurement were employed to investigate the shock interaction phenomena.

\section{Experimental setup}

\subsection{Ballistic range facility}

The experiments were conducted in a ballistic range employing a single-stage powder gun operation mode at the Institute of Fluid Science, Tohoku University. The ballistic range facility possesses the ability to generate various pressure waveforms by launching a projectile, enabling the investigation of the effects of the turbulence on a long rise-time pressure signature. In this facility, three operation modes: two-stage light-gas gun, single-stage powder gun, and single-stage light-gas gun modes, are available to launch a projectile at an arbitrary velocity [33, 34]. In the present experiment, the singlestage powder gun mode, the ability to launch a projectile $15 \mathrm{~mm}$ in inner diameter at a velocity up to $2.0 \mathrm{~km} / \mathrm{s}$, was employed (Fig. 1). The ballistic range consists of a propellant chamber, a launch tube $15 \mathrm{~mm}$ in inner diameter and $3 \mathrm{~m}$ in length, and a recovery tank $1.66 \mathrm{~m}$ in inner diameter and $12 \mathrm{~m}$ in length. There are three optical window pairs for the flow visualization. The launch tube with a projectile at its tube end is connected to the propellant chamber. A rifle cartridge (Winchester 300) filled with a smokeless powder (HS-7, Hodgdon Powder Co.) is ignited with a detonator (GM210M, Federal Gold Medal Match), and then a smokeless powder (H4350, Hodgdon Powder Co.) is ignited, which results in the acceleration of the projectile due to the high-pressure gas. The amount of the H4350 smokeless powder was adjusted depending on the mass of the projectile in other to launch the projectile at the required velocity. A blast tube $15 \mathrm{~mm}$ in inner diameter and $1.6 \mathrm{~m}$ in length was connected at the launch tube end, making it effective to attenuate the precursory blast waves driven in front of the projectile. The experimental setup in the recovery tank is illustrated in Fig. 2. The projectiles flew from the left side to the right side. While the projectiles traveled through the blast tube, a trigger signal was captured as they passed a laser beam for the measurements. The launched projectile 
was stopped at a catch tank after the pressure and the density fields were measured.

\subsection{Projectiles}

To simulate the sonic boom signature, near-field pressure waveforms generated by the cylindrical and cone-cylinder projectiles in supersonic flight were employed. An N-shaped pressure waveform was produced by the cylindrical projectile that was $15 \mathrm{~mm}$ in outer diameter, $70 \mathrm{~mm}$ in length, $15 \mathrm{~g}$ in mass, and made of polyamide resin (Fig. 3.2 (a)). This projectile was useful to visualize a distorted shock wave because of the strong density change around it. The cone-cylinder projectile $15 \mathrm{~mm}$ in outer diameter and $177 \mathrm{~mm}$ in length (Fig. 3.2 (b)) simulated a long rise-time pressure signature, which is a low sonic boom, at the especially near-field of the projectile. This projectile $50 \mathrm{~g}$ in mass comprised two different materials: polyamide resin and steel. Although the steel part prevents the deformation of the nose by aerodynamic forces, to avoid damage of the launch tube, we did not use the steel material on the surface that was in contact with the launch tube. Figure 4 shows the dimensions of the conecylinder projectile. Carlson et al. [35] defined the angle of the cone, and reported that the shape of the cone produced a near-field pressure waveform with a long rise time. In the present design, the projectile has a second ramp that generates a pressure signature with a short rise time, at the connection part. The cone-cylinder projectile that simultaneously produces the long and short rise-time pressure signatures was used to compare the effects of the turbulence on the long and short rise-time pressure signatures. A Bridgman seal manufactured at the rear edge of the projectile prevents the leakage of the combustion gas and leads to a high launching repeatability. In the present experiment, the launching projectile velocity was set at a Mach number of 1.4 .

\subsection{Near-field pressure measurement}

The pressure waveforms were measured at two locations using a flush-mounted piezoelectric pressure transducer (PCB Piezotronics, Inc. model: 113B28, rise time: $\leq 1 \mu$ s, resolution: $7 \mathrm{~Pa}$, resonant frequency: $\geq 500 \mathrm{kHz}$ ) in each shot. To prevent the mechanical oscillation from affecting the pressure waveforms, the pressure transducer was screwed to a mount holder made of MC nylon. Ukai et al. [36] showed that a pressure transducer must be positioned at a distance from the edge of the flush-mounting plate to avoid the effects of shock diffraction from the edge. Thus, the mount holder was embedded at the center of a steel plate $250 \mathrm{~mm} \times 400 \mathrm{~mm} \times 15 \mathrm{~mm}$ (length $\times$ width $\times$ thickness) in size, which results in an accurate pressure measurement. Two steel plates with the pressure transducer were placed at distances of 1400 and $1700 \mathrm{~mm}$ from the blast tube end, and these pressure measurement locations are defined as P1 and P2, respectively (Fig. 2). The pressure waveforms affected by the turbulence 
were measured at P1. The pressure transducers were positioned at a height of $140 \mathrm{~mm}$ under the flight path, and their angle was adjusted so that the wavefront of the shock wave is in contact with the pressure transducer and the steel plate; i.e., the angle is the same as a Mach angle (Fig. 5). The pressure signals was recorded using a digital oscilloscope (Yokogawa Electric Corp., model: DL750, resolution: 16 bit) via a low-noise signal conditioner (PCB Piezotronics, Inc. model: 482A22). The sampling rate was set as $10 \mathrm{MS} / \mathrm{s}$ in order to accurately measure the pressure waveform.

\subsection{Turbulence impingement}

The pressure wave was passed through a turbulent field generated by a circular jet nozzle $4.5 \mathrm{~mm}$ in inner diameter. The end of the jet nozzle was positioned in front of the pressure transducer P1, separated by $45 \mathrm{~mm}$ vertically and $110 \mathrm{~mm}$ horizontally, and air was blown parallel to the plate surface (Fig. 5). High-pressure dry air supplied through a pressure regulator and a flexible tube was used as the jet gas. To control the jet velocity, the static pressure at the nozzle end was maintained at $60.0 \pm$ $0.6 \mathrm{kPa}$. The static pressure was measured using a pressure transducer (Honeywell, HSC Series, pressure range: 0 - $100 \mathrm{kPa}$, measurement error: $\pm 1 \%$ FSS) during the shot.

To evaluate the turbulent field, a hot-wire anemometer (Institute of Flow Research Corp., model: CTA-002) with an X-probe (KANOMAX Corp., model: 0252R-T5) was used. The voltage signals from the hot-wire anemometer were recorded by the Yokogawa oscilloscope at a sampling rate of 100 $\mathrm{kHz}$ with a recording time of $2 \mathrm{~s}$. The signals were calibrated before the turbulence measurement. A pitot-tube anemometer that was connected to a digital manometer (COSMO Instrument Co., Ltd, model: DM-3501) is positioned next to the hot-wire anemometer. Since the non-uniform velocity distribution generally appears in the jet turbulent field, the pitot tube was immediately exchanged with the hot-wire's position for the calibration, and the mean velocity was measured by using the pitot tube as a reference. The voltage signals from the digital manometer was recorded using a compact DAQ system (National Instruments Corp., model: 9205) driven by LabVIEW 2011. When the turbulent field was evaluated, the pitot tube and the steel plate were removed.

\subsection{Visualization techniques}

High-speed schlieren photography and a point-diffraction interferometer (PDI) were employed to visualize a distorted shock wave by impinging the turbulence. The interaction behavior is observable at the pressure measurement position P1: a parallel beam is passed through the optical window pair equipped near the blast tube end. Unsteady flow was successfully visualized using a PDI optical system in a previous study [37]. A pair of parabolic mirrors $300 \mathrm{~mm}$ in diameter with a focal length of 2800 
$\mathrm{mm}$ and a pair of flat mirrors $550 \mathrm{~mm}$ in diameter were used. A light was supplied from a continuouswave green laser (Coherent Inc., model: COMPASS 315M-50, wavelength $=532 \mathrm{~nm}$, output power $=$ $50 \mathrm{~mW}$ ). The laser beam expanded by a concave lens was collimated by the parabolic mirror, and underwent a change in direction owing to the flat mirror. The collimated light passed through the test section via the optical glass windows and was then reflected by the second flat and parabolic mirrors. The offset angle between the collimated light beam and the light source was the same at the first and second mirrors to prevent coma. To obtain an interference pattern, a small pinhole, installed on a semitransparent plate, was positioned at the laser focal point of the second parabolic mirror. The converging light is diffracted when passing through the pinhole, and it spreads semi-spherically. This diffracted light acts as a reference beam and overlaps with the object beam, forming an interference pattern [38, 39]. A $290 \mathrm{~W}$ continuous light source (metal halide lamp LS-M210, Sumita Optical Glass, Inc.) was used in the schlieren-photography mode as a substitute for the green laser, and a horizontally positioned knife edge was replaced with the pinhole plate. Moreover, a diffuser panel, a convex lens, and a pinhole were replaced with the concave lens positioned in front of the green laser source. Visualization images were recorded using a high-speed digital camera (Shimadzu Corp., model: HPV-X, 128 frames at 400 $\times 250$ pixels spatial resolution). A frame rate of $200 \mathrm{kfps}$ with an exposure time of $0.2 \mu \mathrm{s}$ (PDI mode) and $0.5 \mu$ s (schlieren mode) was used.

\section{Results and discussion}

\subsection{Turbulent field}

An almost symmetric turbulent field was generated from the circular nozzle although it did not correspond with the characteristics observed under the real atmospheric turbulence. Figures 6 and 7 show the mean nozzle velocity $\bar{U}$ in the $x-y$ plane and the distribution of the turbulence characteristic $v_{r m s} / \bar{U}_{110}$ of the y-component in the x-y plane, respectively. Where, the overline and subscript " 110 " denote the time average and the velocity at coordinate $(x=110, y=0, z=0)$, respectively. A righthanded coordinate system is employed (Fig. 5). The turbulence characteristic is defined as the rootmean-square velocity fluctuation of the $\mathrm{x}$-component divided by its mean velocity at coordinate (110, $0,0)$. The fluctuation velocity is obtained from $v=V-\bar{V}$, and the root-mean-square velocity

fluctuation is defined as $v_{r m s}=\sqrt{\overline{v^{2}}}$. In this paper, only the velocity fluctuation of the y-component is considered because a flow disturbance in the same direction as the shock propagation strongly affects a pressure waveform [28, 40]. The dotted lines shown in Figs. 6 and 7 indicate a shock propagation path that reaches to the pressure transducer, and the shock wave passed through a turbulent field 
approximately $40 \mathrm{~mm}$ in length. Since the turbulent field was generated away from the pressure transducer, a weak pressure wave induced by the jet turbulence did not disturb the pressure signal from the pressure transducer; of course, we checked this before the shot. At coordinate $(110,0,0)$, the mean velocity of the x-component was $\bar{U}_{110}=62.2 \mathrm{~m} / \mathrm{s}$ with the Reynolds number of $1.9 \times 10^{4}$ based on the jet nozzle's inner diameter, and the turbulence characteristic of the y-component was $v_{r m s} / \bar{U}_{110}$ $=0.19$.

The almost ideal turbulent field appeared at off-center from the nozzle axis although the full turbulent field was not able to be developed in the present experimental setup. We evaluated the turbulence spectra on the shock propagation path that reaches to the pressure transducer. Based on the hot-wire measurement data, the one-dimensional spatial energy spectra of the turbulence at coordinates $(110,-20,0),(110,-10,0)$, and $(110,0,0)$ were obtained (Fig. 8). A dotted line indicates Kolomogorov's $-5 / 3$ power law which can discern the inertial sub-range that is unaffected by the shear flow and the molecular viscosity $[41,42]$. The one-dimensional spatial energy spectra were obtained as the following process; the frequency-domain power spectrum of the velocity fluctuations $P(f)$ obtained by FFT was used to calculate its energy spectrum $E(f)=2 \pi P(f)$. The frequency domain were converted into a spatial domain defined as the wavenumbers $K=(2 \pi f) / \bar{U}$ using Taylor's hypothesis [42], and then a spatial energy spectrum $E(K)=E(f)(\bar{U} / 2 \pi)$ was obtained. At coordinate $(110,0$, 0 ), the shear flow may strongly affect the energy cascade which transfers an energy from a large scale vortex to a small scale vortex because the spectrum curve does not fit the gradient of Kolomogorov's $-5 / 3$ power law. The energy cascade at the high wavenumber range seems to be affected by the shear flow strongly. However, at coordinates $(110,-10,0)$ and $(110,-20,0)$ which are off-center from the nozzle axis, the energy cascade is almost unaffected by the shear flow and the molecular viscosity.

Scaling the spatial and time scale characteristics which appears in the real sonic boom propagation is important for model experiments, and the comparison with the original scale characteristics makes it possible to clearly show the experimental conditions. Based on the hot-wire measurement data, we estimated the spatial length scales. To obtain the outer length scale $L_{o}$, the outer time scale $\epsilon$ at coordinate $(110,-20,0)$ where the ideal turbulent field appeared was computed using Equation (1).

$$
\epsilon=\int_{0}^{\tau_{1}} C(\tau) d \tau, \quad C(\tau)=\frac{\overline{u(t) u(t+\tau)}}{\overline{u^{2}(t)}}
$$

The quantity $\tau$ denotes time interval, and the autocorrelation coefficient $C(\tau)$ becomes to zero at the position of time interval $\tau_{1}$. The outer length scale was defined as $L_{o}=\bar{U} \epsilon$ estimated using Taylor's hypothesis. The inner length scale $L_{i}$ which is a turbulence parameter was defined as the following Equation (2). 


$$
L_{i} \equiv\left(\frac{v^{3}}{\varepsilon}\right)^{\frac{1}{4}}, \quad \varepsilon=2 v \int_{0}^{\infty} K^{2} E(K) d K
$$

$v$ is the kinetic viscosity, and $\varepsilon=2.16 \times 10^{4} \mathrm{~m}^{2} / \mathrm{s}^{3}$ was the energy dispersion of the $\mathrm{x}$-component at coordinate $(110,-20,0)$. Table 1 presents the typical spatial scale characteristics: shock propagation distance, the thickness of the turbulent field, the outer length scale, and the inner length scale. In the present experiment, the scaling factor of approximately 1/50000 is obtained although the inner scale has the different scaled order. The scaling about the pressure waveform has not been taken into account in the present study because a long rise-time pressure signature by design has never been observed under sonic boom experiments.

The strong velocity fluctuation compared with that in the real turbulence atmosphere was developed to shorten the distance to a first caustics which induces the shock wave focusing. The time scale characteristics of the turbulence: the wind velocity and the velocity fluctuation, are approximately 4 times as that of the real atmosphere. The wind velocity recorded during the sonic boom experiments was range up to $15 \mathrm{~m} / \mathrm{s}$ [8] and the velocity fluctuation was range up to $2.5 \mathrm{~m} / \mathrm{s}$ [43, 44] were used for the comparison. The strong velocity fluctuation ( $v_{r m s}$ was range up to $11.8 \mathrm{~m} / \mathrm{s}$ ) that generated in the present experiment leads to enhancement of the wave scattering, making it possible to shorten the distance to the first caustics. The distance to the first caustics was estimated using the approximation equation (3) [46] as

$$
y_{\text {caust }} \cong 0.28 L\left(\frac{c_{0}}{v_{\text {rms }}}\right)^{\frac{2}{3}}+y_{\text {travel }}
$$

$y_{\text {travel }} \cong 135 \mathrm{~mm}$ is the distance between the projectile and the turbulent field (the shock propagation distance within a stationary atmosphere), and $c_{0}=342 \mathrm{~m} / \mathrm{s}$ is the sound speed within the recovery tank. The velocity fluctuations of y-component $v_{r m s}$ at coordinate from $(110,0,0)$ to $(110,-20,0)$ were used to evaluate the first caustics at the whole turbulent field because the turbulent field was inhomogeneous in the present experiment. $L$ denotes the characteristic length scales of the turbulence, and the distance to the first caustics depends on the characteristic length scales: the outer and inner length scales. The first caustic caused by the inner length scale: $L=L_{o}$, occurs within the turbulent field, and its distance from the projectile were a range of 146 and $172 \mathrm{~mm}$; i.e., the shock focusing occurs during the shock propagation within the turbulent field $\left(y_{\text {caust }}<175 \mathrm{~mm}\right.$ ). The inner length scale: $L=L_{i}$, also induce the shock focusing within the turbulent field, and the first caustics distance was approximately $135 \mathrm{~mm}$ $\left(y_{\text {caust }}<175 \mathrm{~mm}\right)$. Therefore, the shock focusing occurs in the present experimental condition in spite of the thin turbulent field. 


\subsection{Turbulence interaction with $\mathrm{N}$-shaped signature}

The clearly distorted wavefront of the shock wave arising from the jet turbulence interaction means enabling the investigation of the shock-turbulence interaction in the ballistic range facility. Figure 9 shows continuous schlieren images of the turbulence interaction with the shock waves generated by the cylindrical projectile. A bow shock wave defined as a primary shock occurred in front of the projectile, and then a secondary shock was generated behind the front edge of the projectile. A recompression shock was induced by a wake flow behind the projectile. A sharp primary shock wavefront generated in front of the projectile transformed to a wavy shape while the shock wave interacted with the turbulent field (Fig. 9 (b)). Its deformation was still retained after it passed the turbulent field, and its wavefront impinged on the pressure transducer. To clearly visualize the shock deformation, a magnified image was captured using the PDI with a high sensitivity (Fig. 10). The right and left columns show the cases with and without the turbulence interaction, respectively. The distance between each interference pattern, which denotes the isopycnic line, differed slightly between the images shown in (a) and (h) because the launching velocities were not exactly equal. The interference patterns varied during the shock propagation even if the shock wave does not interact with the turbulence (Figs. 10 (a-g), the white boxed areas). The narrow distance between the adjacent fringes meaning a high pressure magnitude was expanded with time; namely the overpressure magnitude decreased. This is because the pressure magnitude is gradually attenuated when the shock wave propagates away from the projectile. On the other hand, in the case of the shock-turbulence interaction (Figs. 10 (h-o)), the distance did not necessarily broaden while the primary shock traveled in the turbulent field due to the turbulence interaction. The fringes oscillated irregularly, and then several narrow fringes at the primary shock wavefront location appeared, as shown in Fig. 10 (o).

The effects of the turbulence on the pressure waveform were observable although the pressure waveform was not materially distorted. The pressure waveform with an extremely large overpressure magnitude: approximately $\Delta P=30 \mathrm{kPa}$ in the present result, may not yield a strong shock deformation. Figure 11 shows a typical N-shaped waveform pair measured at P1 and P2 in the same shot. Since the jet nozzle was located at P1 only, almost identical pressure signatures were observable at P1 and P2 when the jet flow was switched off. The pressure waveforms were processed by a digital low-pass filter with a cutoff frequency of $120 \mathrm{kHz}$ because the pressure signals oscillated owing to the resonant frequency of the pressure transducer. This digital low-pass filter is a finite-impulse-response type designed using MATLAB R2013a. The gain value of the low-pass filter possess a flat characteristic during a pass frequency. The overpressure denotes the increase in the pressure due to the primary shock. The secondary shock leads to the pressure recovery from the negative pressure to the ambient pressure. 
The recompressed shock has the same capability as the secondary shock. Ideally, when the shock wave propagates in a stationary atmosphere, the pressure waveforms measured at P1 and P2 are identical. However, the free flight projectile's attitude may have slightly, and its velocity decreases owing to the aerodynamic forces acting on it, which results in a different pressure waveform. Although it is difficult to eliminate these undesired effects, an almost uniform waveform was captured (Fig. 11 (a)). On the other hand, in the turbulence interaction case (Fig. 11 (b)), a high overpressure magnitude remained before the pressure rapidly decreased compared with the pressure waveform measured at P2. The waveform measured at the position P1 is related to the PDI images shown in Figs. 10 (h-o); the density and pressure fields were simultaneously captured in the same shot. The pressure peak was slightly altered to a round shape although a high magnitude was maintained because the wavefront of the shock wave with several narrow fringes impinged on the pressure transducer.

\subsection{Turbulence interaction with long rise-time signature}

Long and short rise-time pressure signatures were simultaneously produced by the cone-cylinder projectile. The density fields visualized using the PDI around the cone-cylinder projectile are shown in Fig. 12. The right and left columns show the cases with and without turbulence interaction, respectively. Figure 13 shows the typical long rise-time pressure waveform pairs, each comprising measurements taken at P1 and P2 in the same shot. The measured waveforms were processed by a digital low-pass filter with a cutoff frequency of $350 \mathrm{kHz}$ designed using MATLAB R2013a. A primary shock was generated from the nose of the projectile; however, it was difficult to clearly visualize this owing to the extremely low density change. An isentropic compression wave is generally produced by a conical ramp [47] and increases the pressure with a long rise time. The pressure with a long rise time comprises the pressure field between the primary shock and a primary expansion wave. The primary expansion wave is generated from the end of the conical ramp and induces a pressure drop, and then the pressure rapidly increases because of the secondary shock generated from the ramp of a connection part between the two different materials: polyamide resin and steel. The secondary shock possesses the short rise-time pressure signature. After the pressure is decreased by a secondary expansion wave, a tertiary shock from the Bridgman seal yields a slight pressure increase, and then the decreased pressure due to a tertiary expansion wave increases because of a recompression shock.

The secondary shock with the short rise-time pressure signature was materially distorted compared with that for the long rise-time pressure signature. When the projectile flew in the stationary atmosphere, the sharp wavefronts of the shock remained (Figs. 12 (a-f)), yielding a good correspondence between the waveforms measured at P1 and P2 (Fig. 13 (a)). On the other hand, in the 
case of the shock-turbulence interaction, the wavefront of the secondary shock was distorted by the turbulence (Figs. 12 (j-m)). A comparison between the waveforms measured at positions P1 and P2 indicates that the overpressure due to the secondary shock was strongly attenuated by the turbulence (Fig. 13 (b)). Although the turbulence effects are apparent, the pressure waveform in the long rise-time part, the pressure between the primary shock and the primary expansion wave, was not materially distorted compared with the waveform having the short rise time.

Regarding the long rise-time pressure signature, the apparent turbulence effects was not observed under the present experimental conditions. In the ballistic range facility, it was difficult to repeatedly produce exactly the same overpressure magnitude. To eliminate the effects of the variation of the overpressure magnitude between the shots, the relative overpressure was obtained using the pressure waveforms measured at P1 and P2 for the same shot. The flight height was estimated according to the spatial flight trajectory determined by a bullet mark on a target plate. A polyethylene terephthalate sheet was placed at a distance of $2500 \mathrm{~mm}$ from the nozzle end for the target plate. A line between the center of the muzzle and the center of the impact scar was considered as the flight trajectory. The difference in the flight height between the measurement positions P1 and P2 was $\widehat{\Delta h}=0.8 \pm 0.6 \mathrm{~mm}$, and the maximum difference was $\Delta h_{\max }=2.0 \mathrm{~mm}$, where the circumflex denotes the ensemble average. Therefore, the effect of the flight height is not a critical issue regarding the relative overpressures obtained for the same shot. Figure 14 shows the relative overpressures $\Delta P 1_{s} / \Delta P 2_{s}$ and $\triangle P 1_{p} / \Delta P 2_{p}$, and Table 2 presents the mean and standard deviation. The suffixes 1 and 2 denote the positions P1 and P2, respectively. The subscripts $s$ and $p$ indicate "secondary shock" and "primary shock," respectively. The abscissa axis shows the number of shots, which is randomly selected. Twenty and seven projectiles were shot in the cases with and without the turbulence interaction, respectively. Since the flight speed of the projectile is slightly decreased generally by the aerodynamic forces acting on the projectile, the overpressure magnitude at $\mathrm{P} 1$ becomes larger than that at $\mathrm{P} 2$. Thus, the mean relative overpressure magnitudes greater than 1.0 occurred even though they were measured without the turbulence interaction. For the primary shock (Fig. 14 (a)), the mean and standard deviation hardly changed even though the jet turbulence impinged on the primary shock; the standard deviation increased by a factor of 1.5 in the turbulence interaction case. For the secondary shock (Fig. 14 (b)), an extremely wide dispersion occurred with the turbulence, and there was a small dispersion without the turbulence. The standard deviation increased by a factor of 14 owing to the turbulence interaction. A comparison of the relative overpressures between the primary and secondary shocks clearly indicates that the relative overpressure of the secondary shock was strongly affected by the turbulence.

The pressure waveform of the long rise-time signature slightly altered by the turbulence interaction 
although it was not apparent turbulence effects. To evaluate the weak turbulence effects, a subtracted waveform, which is obtained as pressure differential between the waveforms measured at P1 and P2, was computed as $p_{s u b}=p 1-p 2$. The subtracted waveforms of the typical shots I , II , and III are shown in Fig. 15, and these shot numbers correspond with the symbols shown in Fig. 14. In the case without the turbulence interaction (Fig. 15 (a), shot I ), the subtracted pressure gradually increases at a range of approximately $0.08-0.18 \mathrm{~ms}$ because the flight speed of the projectile slightly decreased. The subtracted pressure decreases at approximately $0.2 \mathrm{~ms}$ which the secondary shock wave arrives because the secondary shock arrival time measured at P2 was faster than that measured at P1 resulting from the slight Mach angle increase. In the case with the turbulence interaction (Fig. 15 (b) and (c), shots II and III), the subtracted waveform has some large round shapes at a range of approximately 0 to $0.18 \mathrm{~ms}$. It seems that the shock focusing/diffracting induce the round shape. The subtracted pressure is decreased at a range of $0.13-0.18 \mathrm{~ms}$ by the shock focusing/diffracting in spite of the subtracted pressure increase owing to the flight speed decrease. The subtracted pressure decrease at a range of $0.13-0.18 \mathrm{~ms}$, corresponds with the relative overpressure of the primary shock decrease (Fig. 14 (a), shots II and III), and the relative overpressures are almost 1.0.

Although the shock focusing/diffracting occurred during the shock wave propagation within the present turbulent field, these effects did not give the same contribution to the short and long rise-time pressure waveforms. In the laboratory-scale experiments which simulate the $\mathrm{N}$-shaped waveform, a front and tail shock waves have the same waveform deformed by turbulent effects [10, 30]. It seems that the front and tail shock waves receive the same shock focusing/diffracting effects. This is because the wave duration time is in the order of $10 \mu \mathrm{s}$, and the vortexes cannot move during the wave duration time. In the present experiment, time differential of the pressure peaks between the primary and secondary shocks was approximately $20 \mu \mathrm{s}$ which is the same as the wave duration time of the laboratory-scale experiments in the order of magnitude. Moreover, the time differential is shorter than the outer time scale of the velocity fluctuation at coordinate $(x=110, y=0, z=0)$. In spite of the short time differential, the shock focusing/diffracting effects did not give the same contribution to the overpressures of the primary and secondary shocks. To evaluate the relation of the turbulence effect between the relative overpressures of the primary and secondary shocks, the spearman rank correlation $r_{s}$ was computed using Equation (4), and $r_{s}=0.3$ which indicates the non-correlation was obtained.

$$
r_{s}=1-\left(\frac{6 \sum d_{i}^{2}}{n\left(n^{2}-1\right)}\right)
$$

where, $d_{i}$ denotes subtraction of rank between the relative overpressures of the primary and secondary 
shocks. $n=20$ is the number of the shot pair. This non-correlation corresponds to the subtracted pressure waveform shown in Fig. 15. In the shot II (Fig. 15 (b)), the subtracted pressure of the secondary shock increased at approximately $0.2 \mathrm{~ms}$; however, the subtracted pressure of the primary shock at approximately $0.18 \mathrm{~ms}$ decreased compared to that in the case without the turbulence interaction; i.e., the shock focusing/diffracting gave the different contribution to the primary and secondary overpressures. However, the long rise time pressure waveform receives the same contribution from the shock focusing/diffracting because the characteristic of the subtracted waveforms persists at a region of 0 to $0.18 \mathrm{~ms}$. On the other hand, in the shot III (Figs. 14 and 15(c)), both of the subtracted pressures at approximately 0.18 and $0.2 \mathrm{~ms}$ decreased compared to that in the case without the turbulence interaction. Note that the subtracted pressure of the secondary shock drops after the subtracted pressure increases because the secondary shock arrival time was changed by the turbulence interaction. Therefore, the shock focusing/diffracting effects did not give the same contribution to the primary and secondary overpressures in spite of the shock wave propagation within the same turbulent field. The shock focusing/diffracting generated in the present turbulent field leads to the deformation of the pressure waveform, and the deformed waveforms of the primary and secondary shocks appeared. However, the contribution of the shock focusing/diffracting effects is different. The primary and secondary shocks have the long and short shock front rise times, respectively. It seems that the shock focusing/diffracting effects depend on the length of the rise time.

\section{Conclusion}

An effect of turbulence interaction on a long rise-time pressure signature was experimentally investigated in a ballistic range facility. To compare the effects of the turbulence on the long and short rise-time pressure signatures, a cone-cylinder projectile that simultaneously produces these pressure signatures was designed. Additionally, to evaluate the experimental setup in the ballistic range facility, the turbulence effects on an $\mathrm{N}$-shaped wave were investigated using a cylindrical projectile that yields the strong density change around it; thus, it is easy to clearly visualize a shock deformation. The turbulence effects were evaluated using flow diagnostic techniques: high-speed schlieren photography, a PDI, and a pressure measurement.

The shock-turbulence interaction can be investigated in the present experimental setup. The effect of the turbulence on the normal $\mathrm{N}$-shaped pressure waveform was observed as the wavefront of its shock wave was distorted when the shock wave passed through the turbulent field. The interference fringes oscillated irregularly because of the turbulence interaction, and the several narrow fringes yielded a pressure peak with a slight round shape although high magnitude was maintained. 
The pressure waveform of the long rise-time signature slightly altered by the turbulence interaction although it was not apparent turbulence effects. The measured pressure waveform exhibited long and short rise-time signatures were used to compare the effects of the turbulence on them. To eliminate the effects of the variation of the overpressure magnitude between the shots, the relative overpressure was obtained using the pressure waveforms measured at two positions in the same shot. For the long risetime pressure signature, the relative overpressure was almost uniform, and its standard deviation 1.5 times greater than that of the no-turbulence case was observed. By contrast, the relative overpressure regarding the short rise time was widely dispersed; the standard deviation increased by a factor of 14 because of the turbulence interaction. Although the strong turbulence effects was not observed in comparison with that of the short rise-time pressure signature, the shock focusing/diffracting, which occurs in the present turbulent field, affected the long and short rise-time pressure signatures. However, the shock focusing/diffracting effects did not give the same contribution to the overpressures of the long and short rise-time signatures. Therefore, this result deduces that the length of the rise time is important to the shock focusing/diffracting effects.

\section{Acknowledgment}

The authors are grateful to the technical staff of the Institute of fluid science, Tohoku University, especially Mr. Toshihiro Ogawa, for the operation of the ballistic range. We also appreciate Mr. Masataka Honna and Mr. Hidenori Ojima for the assistance with the manufacturing. The financial support of the Japan Aerospace Exploration Agency (JAXA) and the Boeing higher education program are also greatly appreciated. 


\section{References}

1. International Civil Aviation Organization, Committee on Aviation Environmental Protection, 7th Meeting, CAEP/7-IP/18 (2007).

2. R. Cowart, "Developing noise standards for future supersonic civil aircraft," In Proceedings of Meetings on Acoustics Vol. 19, 040040 (2013).

3. E.J. Kane, "Some effect of the atmosphere on sonic boom," Proceedings of Sonic Boom Research, NASA SP-147 (1967).

4. J.P. Hodgson, "Vibrational relaxation effects in weak shock waves in air and the structure of sonic bangs,” J. Fluid Mech, Vol.58 (1), pp. 187-196 (1973).

5. H.E. Bass, R. Raspet, "Vibrational relaxation effects on the atmospheric attenuation and rise times of explosion waves,” J. Acoust. Soc. Am. Vol. 64, pp. 1208-1210 (1978).

6. K. Hatanaka, T. Saito, "Numerical analysis of weak shock attenuation resulting from molecular vibrational relaxation," Shock Waves Vol. 21, pp. 121-129 (2011).

7. T.A. Gionfiddo "Analysis of sonic boom data to quantify distortions of shock profiles," NASA conference publication 3172, High-speed research Vol.1, pp. 63-76 (1992).

8. R.A. Lee, J.M. Downing, "Sonic booms produced by United States Air Force and United States Navy aircraft: measured data," AL-TR-1991-0099 (1991).

9. D.A. Hilton, V. Huckel, D.J. Maglieri, "Sonic-boom measurements during bomber training operations in the Chicago area," NASA Technical Note, NASA TN D-3655 (1966).

10. B. Lipkens, D.T. Blackstock, "Model experiment to study sonic boom propagation through turbulence. Part I: General results,” J. Acoust. Soc. Am., Vol. 103 (1), pp. 148-158 (1998).

11. B. Lipkens, D.T. Blackstock, "Model experiment to study sonic boom propagation through turbulence. Part II. Effect of turbulence intensity and propagation distance through turbulence," J. Acoust. Soc. Am., Vol. 104 (3), pp. 1301-1309 (1998).

12. B. Lipkens, "Model experiment to study sonic boom propagation through turbulence. Part III: Validation of sonic boom propagation models," J. Acoust. Soc. Am., Vol. 111 (2), pp. 509-519 (2002).

13. H.S. Ribner, P.J. Morris, W.H. Chu, "Laboratory simulation of development of superbooms by atmospheric turbulence," J. Acoust. Soc. Am., Vol. 53 (3), pp. 926-928 (1973).

14. A. Sasoh, T. Harasaki, T. Kitamura, D. Takagi, S. Ito, A. Matsuda, K. Nagata, Y. Sakai, "Statistical behavior of post-shock overpressure past grid turbulence," Shock Waves Vol. 24 (5), pp.489-500 (2014)

15. J.H. Kim, A. Sasoh, A. Matsuda, "Modulations of a weak shock wave through a turbulent slit jet," Shock Waves, Vol. 20, pp. 339-345 (2010). 
16. A. Niedzwiecki, H.S. Ribner, "Subjective loudness of N-wave sonic booms," J. Acoust. Soc. Am., Vol. 64 (6), pp. 1617-1621 (1978).

17. A.D. Pierce, "Statistical theory of atmospheric turbulence effects on sonic-boom rise times," $J$. Acoust. Soc. Am., Vol. 49 (3), pp. 906-924 (1971).

18. M.A. Averkiou, R.O. Cleveland, "Modeling of an electrohydraulic lithotripter with the KZK equation,” J. Acoust. Soc. Am., Vol. 106 (1), pp. 102-112 (1999).

19. R. Seebass, A.R. George, "Sonic-Boom Minimization,” J. Acoust. Soc. Am., Vol. 51 (2), Part 3, pp. 686-694 (1972).

20. F.E. McLean, B.L. Shrout, "Design methods for minimization of sonic - boom pressure - field disturbances," J. Acoust. Soc. Am. Vol. 39 (5) part. 2, pp. S19-S25 (1966).

21. W.D. Hayes, "Brief review of the basic theory," Sonic Boom Research, NASA SP-147, pp. 3-7 (1967).

22. H.W. Carlson, "Experimental and analytic research on sonic boom generation at NASA," Sonic Boom Research, NASA SP-147, pp. 9-23 (1967).

23. C.M. Darden, B.L. Shrout, "Sonic boom theory: Its status in prediction and minimization," $J$. Aircraft, Vol. 14 (6), pp. 569-576 (1977).

24. J.W. Pawloski, D.H. Graham, C.H. Boccadoro, "Origins and overview of the shaped sonic boom demonstration program," AIAA paper 2005-5 (2005).

25. M.J. Morgenstren, A. Arslan, V. Lyman, J. Vadyak, "F-5 Shaped sonic boom demonstrator's persistence of boom shaping reduction through turbulence," AIAA paper 2005-12 (2005).

26. K.J. Plotkin, J.A. Page, P.G. Coen, D.A. McCurdy, E.A. Haering, J.E. Murray, L.J. Ehernberger, D.J. Maglieri, P.J. Bobbitt, A. Pilon, J. Slamone, "Ground measurements of a shaped sonic boom," AIAA paper 2004-2923 (2004).

27. A.D. Pierce, "Spikes on sonic boom pressure waveforms," J. Acoust. Soc. Am. Vol. 44, pp. 1052 1061 (1968).

28. M. Averiyanov, Ph. Blanc-Benon, R.O. Cleveland, V. Khokhlova, "Nonlinear and diffraction effects in propagation of N-waves in randomly inhomogeneous moving media," J. Acoust. Soc. Am. Vol. 129, pp. 1760-1772 (2011).

29. E. Salze, P. Yuldashev, S. Ollivier, V. Khokhlova, Ph. Blanc-Benon, "Laboratory-scale experiment to study nonlinear N-wave distortion by thermal turbulence," J. Acoust. Soc. Am. Vol. 136 (2), pp. 556-566 (2014).

30. M. Averiyanov, S. Ollivier, V. Khokhlova, Ph. Blanc-Benon, "Random focusing of nonlinear acoustic N-waves in fully developed turbulence: laboratory scale experiment," J. Acoust. Soc. Am. Vol. 130 (6), pp. 3595-3607 (2011). 
31. P.V. Yuldashev, M.V. Averiyanov, V.A. Khokhlova, S. Ollivier, Ph. Blanc-Benon, "Nonlinear Spherically Divergent Shock Waves Propagating in a Relaxing Medium," Acoustical Physics Vol.54 (1) pp. 32-41 (2008).

32. S.V. Hall, "Distortion of the sonic-boom pressure signature by high-speed jets," J. Acoust. Soc. Am. Vol. 63 (6), pp. 1749-1752 (1978).

33. D. Numata, K. Ohtani, M. Anyoji, K. Takayama, M. Sun, "Experimental study of hypervelocity impacts at low temperatures," Shock Waves Vol. 18, pp. 169-183 (2008).

34. T. Ukai, T, Kikuchi, K. Ohtani, S. Obayashi, "Simultaneous visualization of surface and flow field for a projectile" J Visual. Vol.16 (4), pp. 331-340 (2013).

35. H.W. Carlson, R.J. Mack, O.A. Morris, "A wind-tunnel investigation of the effect of body shape on sonic-boom pressure distributions," NASA TN D-3106 (1965).

36. T. Ukai, K. Ohtani, S. Obayashi, "Validation of measurement accuracy for near-field pressure around supersonic projectiles in a ballistic range," Measurement Vol. 67, pp. 24-33 (2015).

37. D. Numata, K. Ohtani, "Application of a point-diffraction interferometer to unsteady shock wave phenomena," Proceedings of 15th International Symposium on Flow Visualization, Belarus (2012).

38. R.N. Smartt, J. Strong, "Point-Diffraction Interferometer," J. Opt. Soc. Am., Vol. 62, pp. 737 (1972).

39. R.N. Smartt, W.H. Steel, "Theory and application of point-diffraction interferometers," Jpn. J. Appl. Phys., Vol. 14, Suppl. 14-1, pp. 351-356 (1974).

40. M.V. Aver'yanov, V.A. Khokhlova, O.A. Sapozhnikov, Ph. Blanc-Benon, R.O. Cleveland, "Parabolic equation for nonlinear acoustic wave propagation in inhomogeneous moving media," Acoust. Phys. Vol. 52, pp. 623-632 (2006).

41. J.O. Hinze, "Turbulence second edition," McGraw-Hill series in mechanical engineering (1975).

42. S. Monin, A.M. Yaglom, Statistical Fluid Mechanics, Vol. II: Mechanics of Turbulence, The MIT press (1975).

43. R. Frehlich, Y. Meillier, M.L. Jensen, B. Balsley, R. Sharman, "Measurements of boundary layer profiles in an urban environment," J. Appl. Meteorol. Vol. 45, pp. 821-837 (2006).

44. I. Smalikho, F. Kopp, R. Rham, "Measurement of atmospheric turbulence by 2-lm Doppler Lidar," J. Atmos. Oceanic Technol. Vol. 22, pp. 1733-1747 (2005).

45. C.M. Sheih, H. Tennekes, J.L. Lumley, "Airborne HotWire Measurements of the SmallScale Structure of Atmospheric Turbulence,” Phys. Fluids Vol. 14 (2), pp. 201-215 (1971).

46. Ph. Blanc-Benon, D. Juve, G. Comte-Bellot, "Occurrence of caustics for high-frequency acoustic waves propagating through turbulent fields," Theoret. Comput. Fluid Dynamics Vol. 2, pp. 271- 
278 (1991).

47. H.W. Liepmann, A. Roshko, "Elements of gasdynamics," Chapter 4, John Wiley and Sons, Inc. (1957). 
Figures

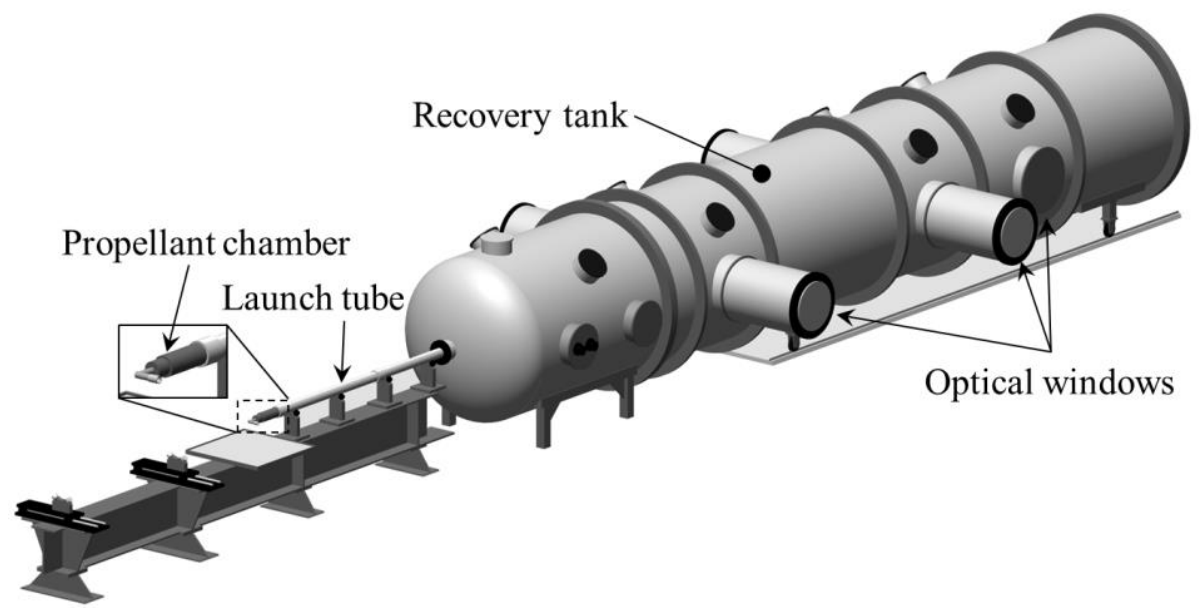

Fig. 1 Schematic of the ballistic range in the single-stage powder gun operation mode at the Institute of Fluid Science, Tohoku University

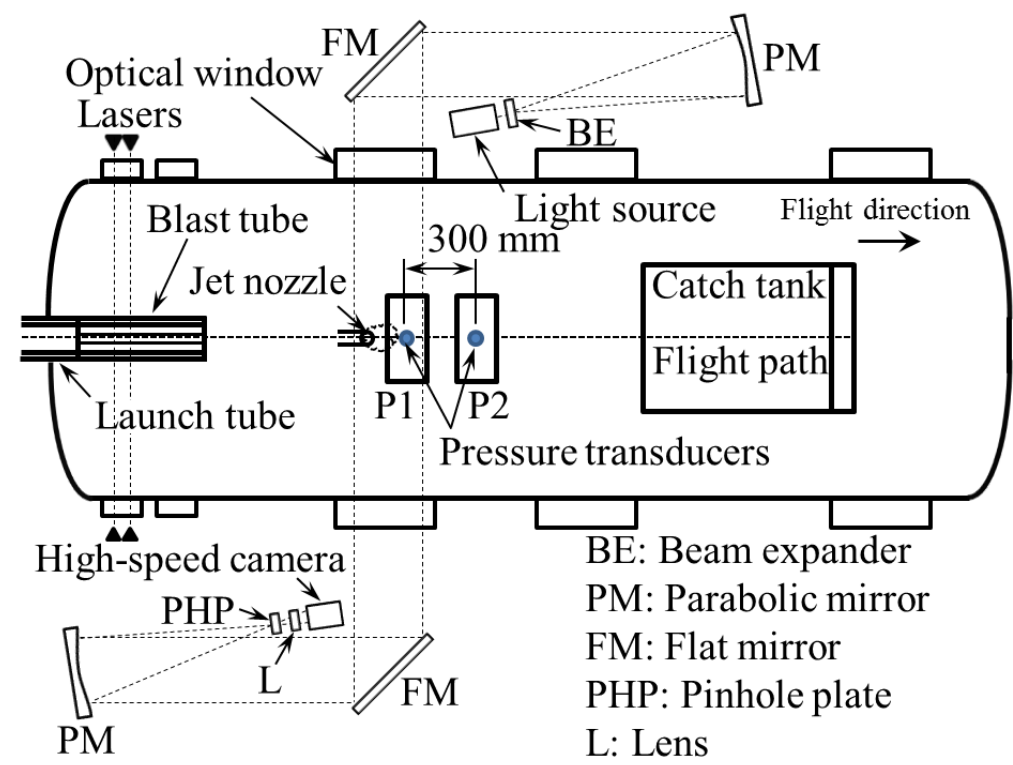

Fig. 2 Experimental setup (the optical arrangement for the PDI)

Polyamide resin

(a) Cylinder type

Polyamide resin

Steel

(b) Cone-cylinder type

Fig. 3 Photographs of the projectiles 


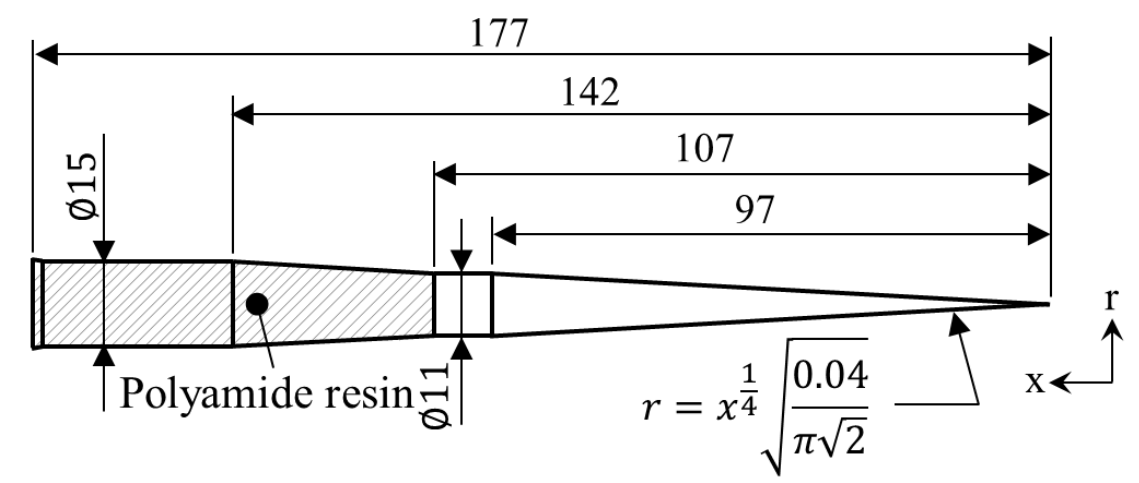

Fig. 4 Model dimensions without the tolerances (units: mm)

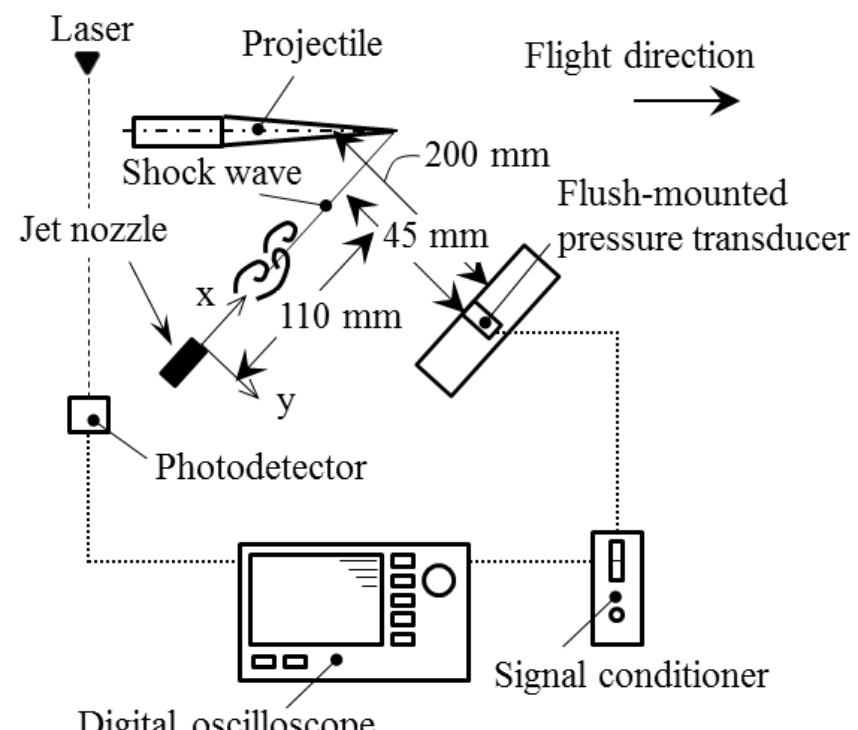

Digital oscilloscope

Fig. 5 Near-field pressure measurement system

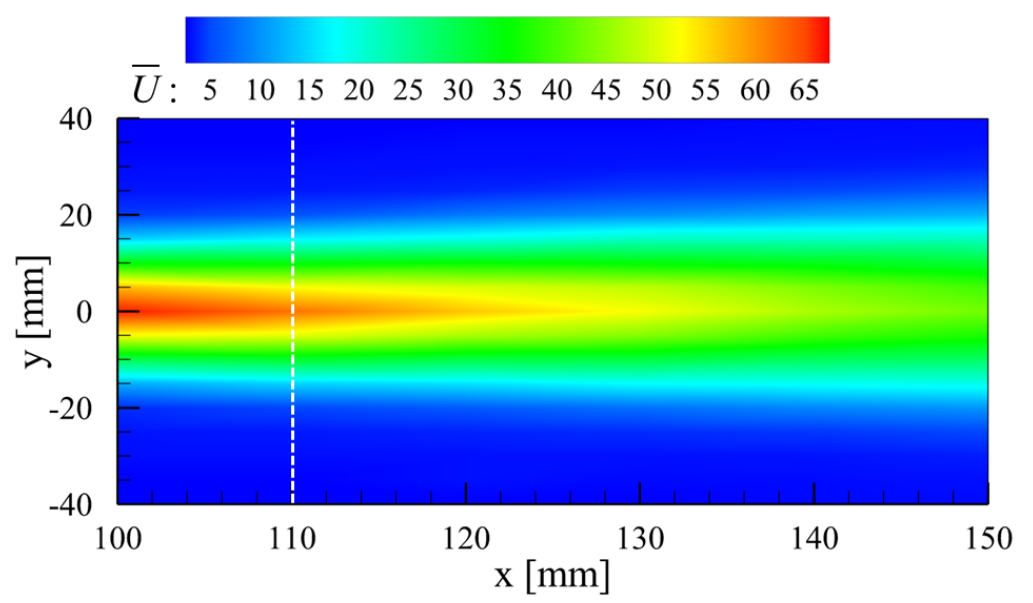

Fig. 6 (Color online) Mean velocity distribution of the $\mathrm{x}$ component 


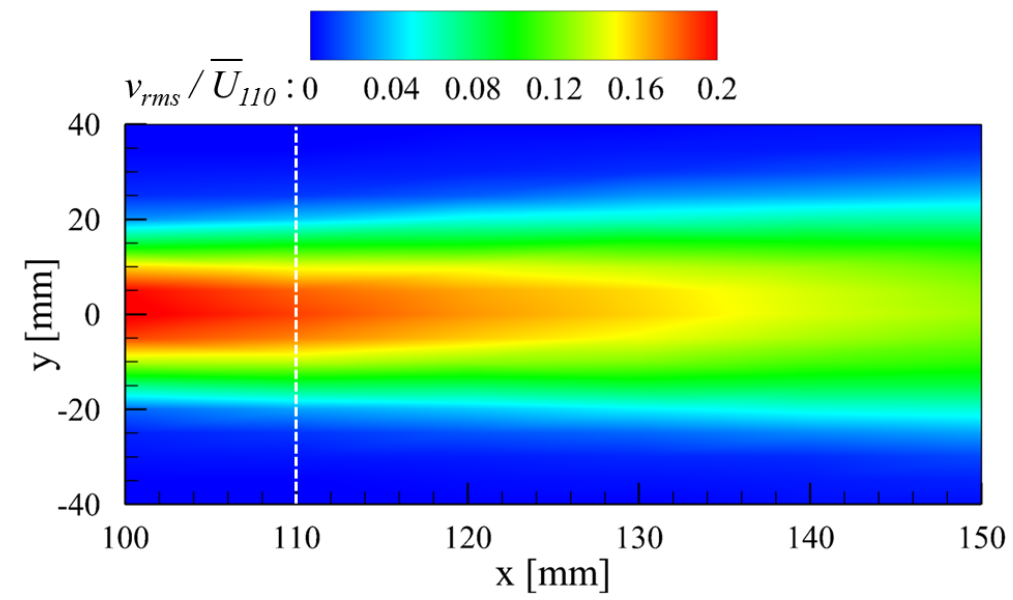

Fig. 7 (Color online) Turbulence intensity distributions of the y component

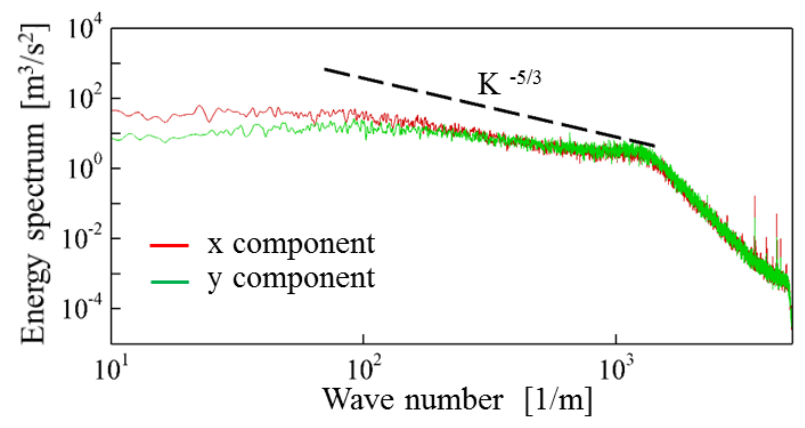

(a) Measured at coordinate $(110,0,0)$

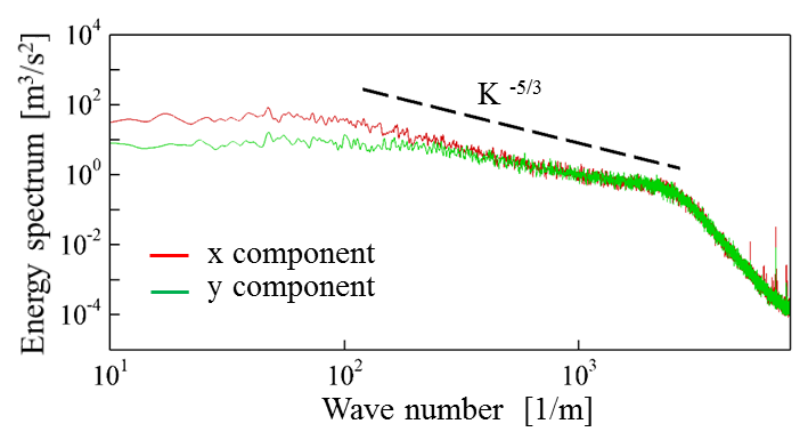

(b) Measured at coordinate $(110,-10,0)$

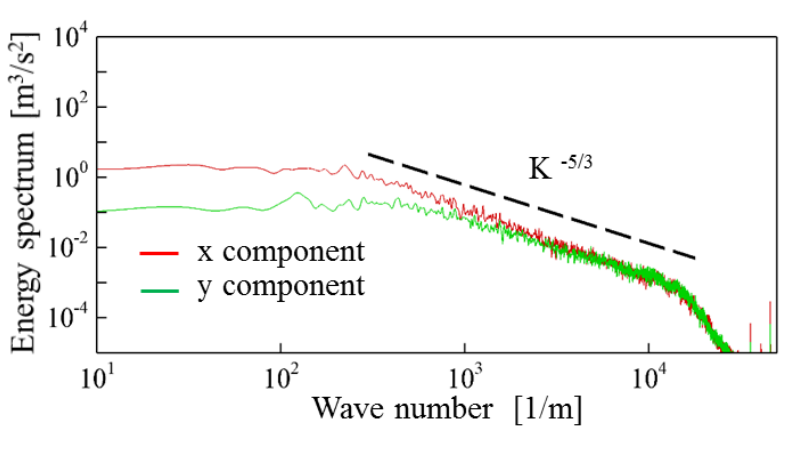

(c) Measured at coordinate $(110,-20,0)$

Fig. 8 (Color online) One-dimensional spatial spectra of the $\mathrm{x}$ and y components. Dotted line: Kolomogorov's $-5 / 3$ power law. 


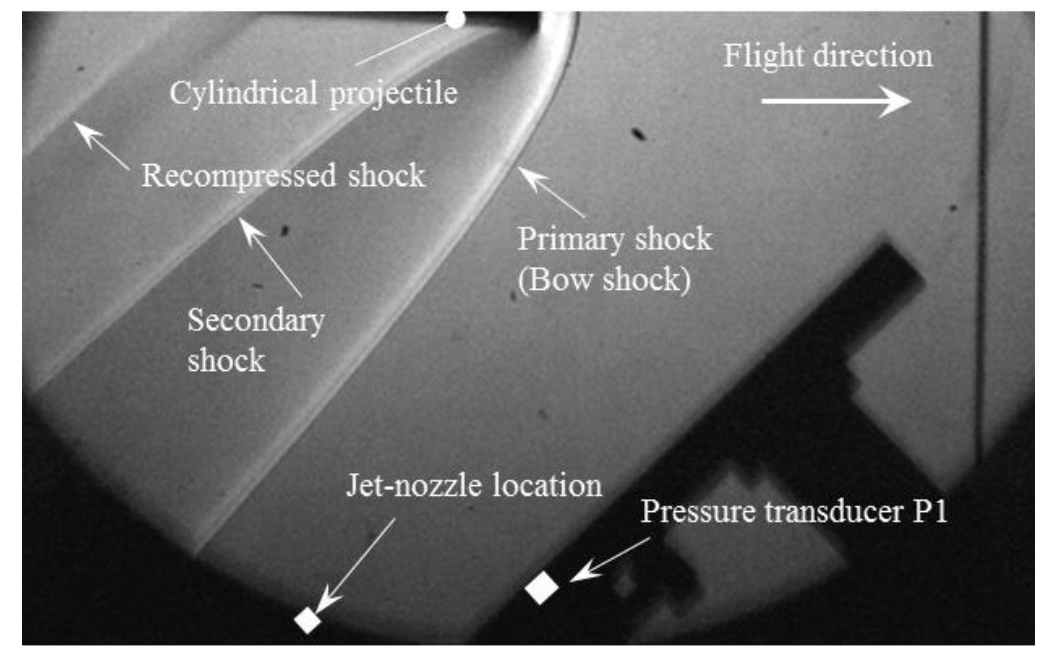

(a) $\Delta t=0 \mu \mathrm{s}$

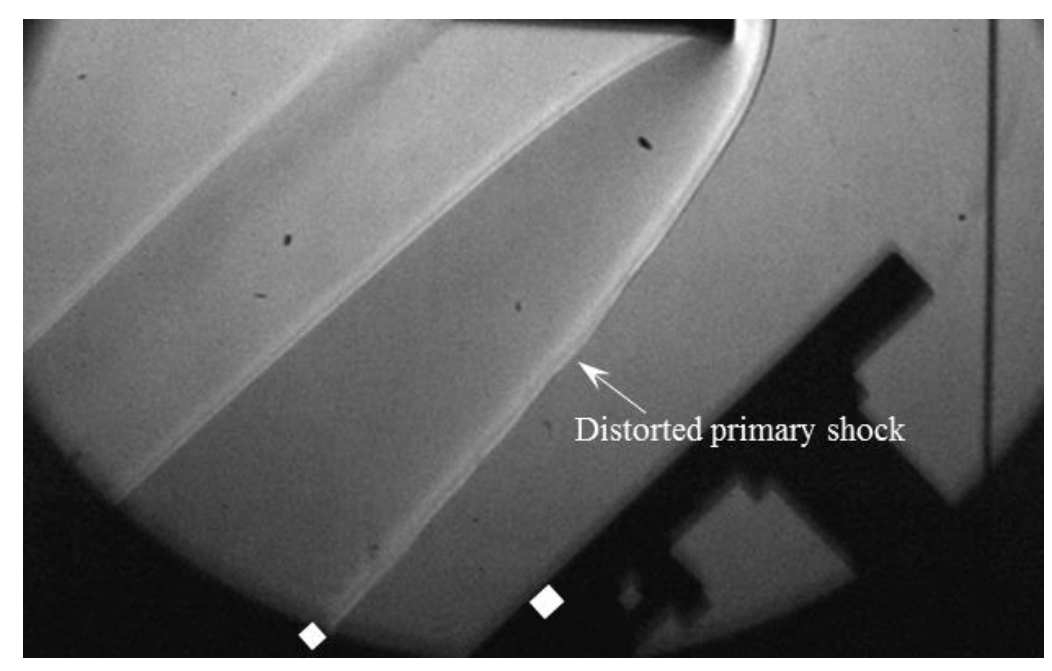

(b) $\Delta t=100 \mu \mathrm{s}$

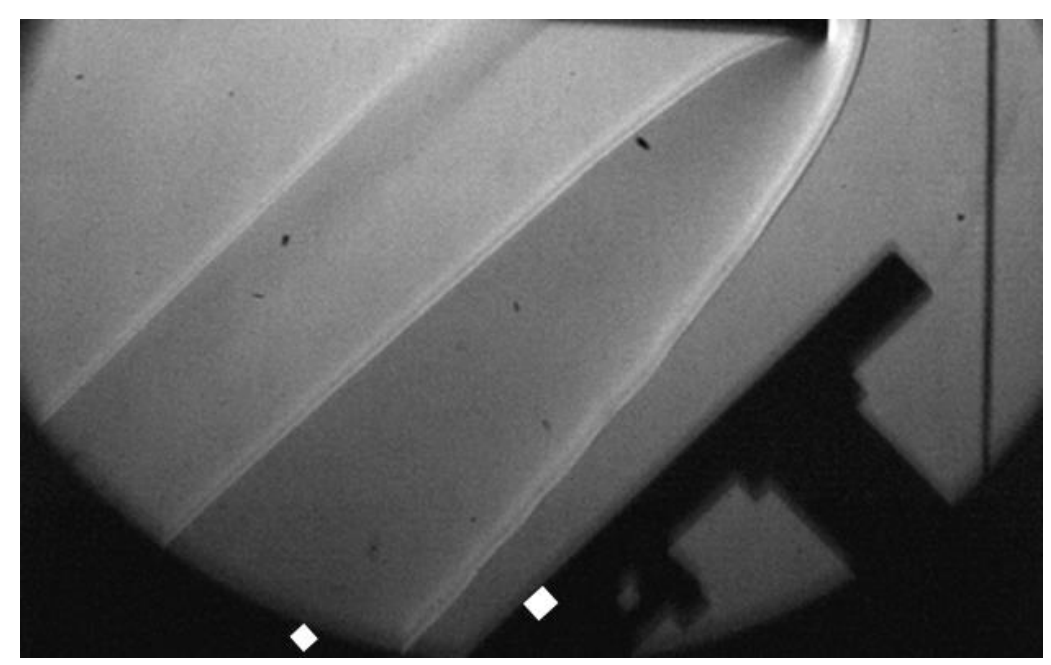

(c) $\Delta t=150 \mu \mathrm{s}$

Fig. 9 Distorted shock waves due to the jet-turbulence interaction: the schlieren images of the cylindrical projectile 


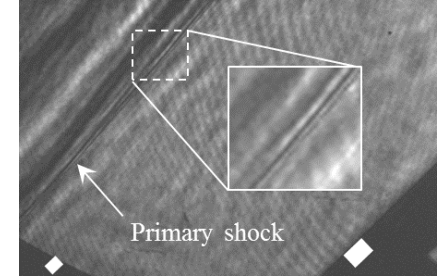

(a) $\Delta t=0 \mu \mathrm{s}$

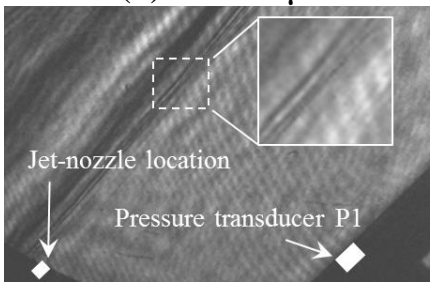

(b) $\Delta t=25 \mu \mathrm{s}$

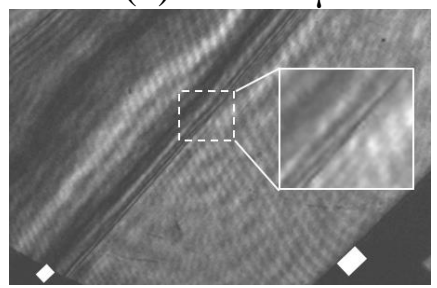

(c) $\Delta t=50 \mu \mathrm{s}$

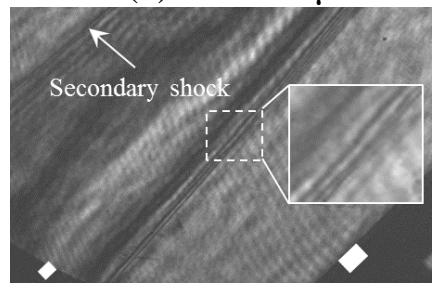

(d) $\Delta t=75 \mu \mathrm{s}$

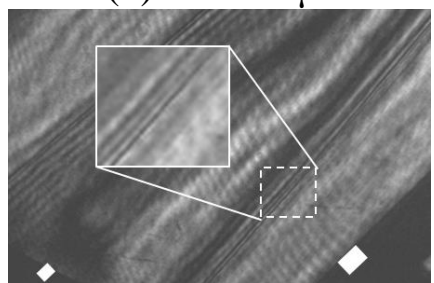

(e) $\Delta t=100 \mu \mathrm{s}$

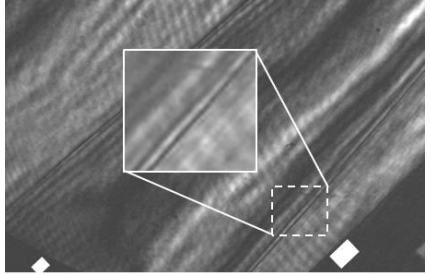

(f) $\Delta t=125 \mu \mathrm{s}$

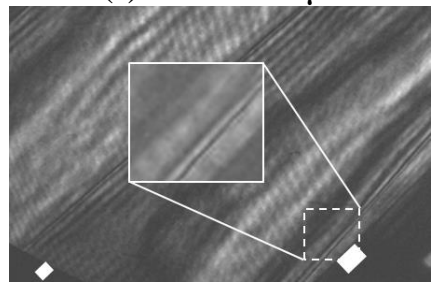

(g) $\Delta t=150 \mu \mathrm{s}$

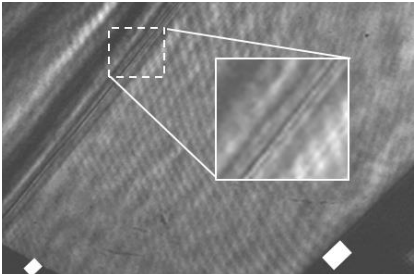

(h) $\Delta t=0 \mu \mathrm{s}$

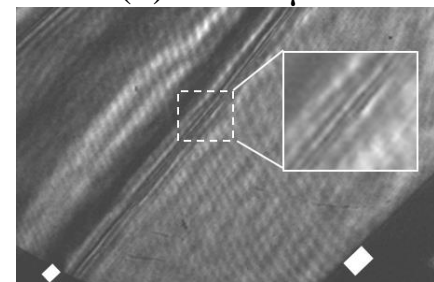

(i) $\Delta t=50 \mu \mathrm{s}$

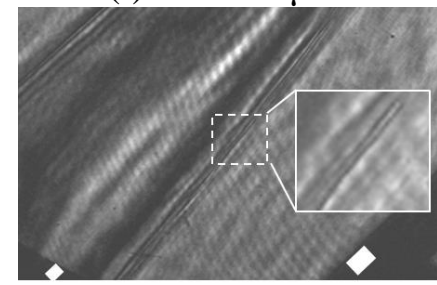

(j) $\Delta t=75 \mu \mathrm{s}$

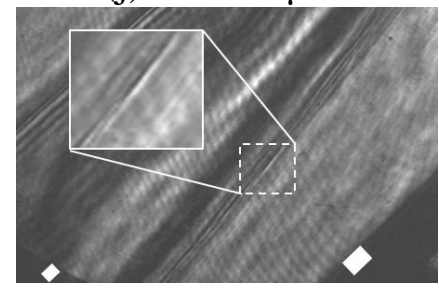

(k) $\Delta t=100 \mu \mathrm{s}$

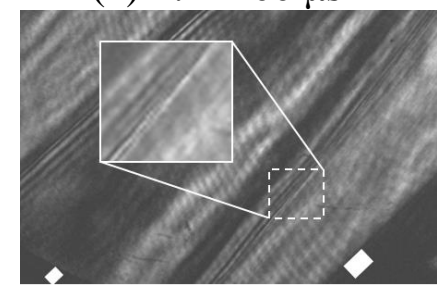

(m) $\Delta t=125 \mu \mathrm{s}$

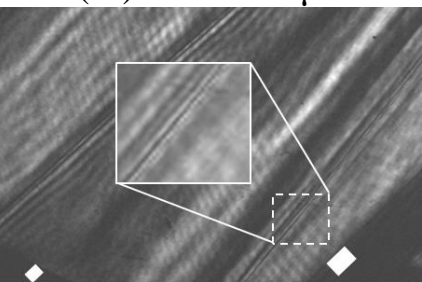

(n) $\Delta t=150 \mu \mathrm{s}$

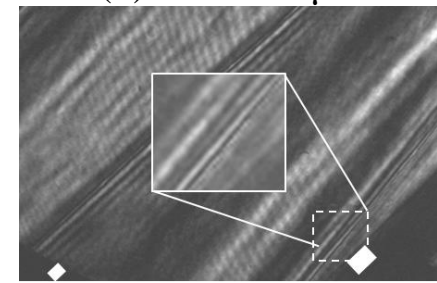

(o) $\Delta t=175 \mu \mathrm{s}$

Fig. 10 Sequential PDI visualization images; the left (a-g) and right (h-o) images correspond to the cases without (shot 1) and with (shot 2) the turbulence interaction, respectively 


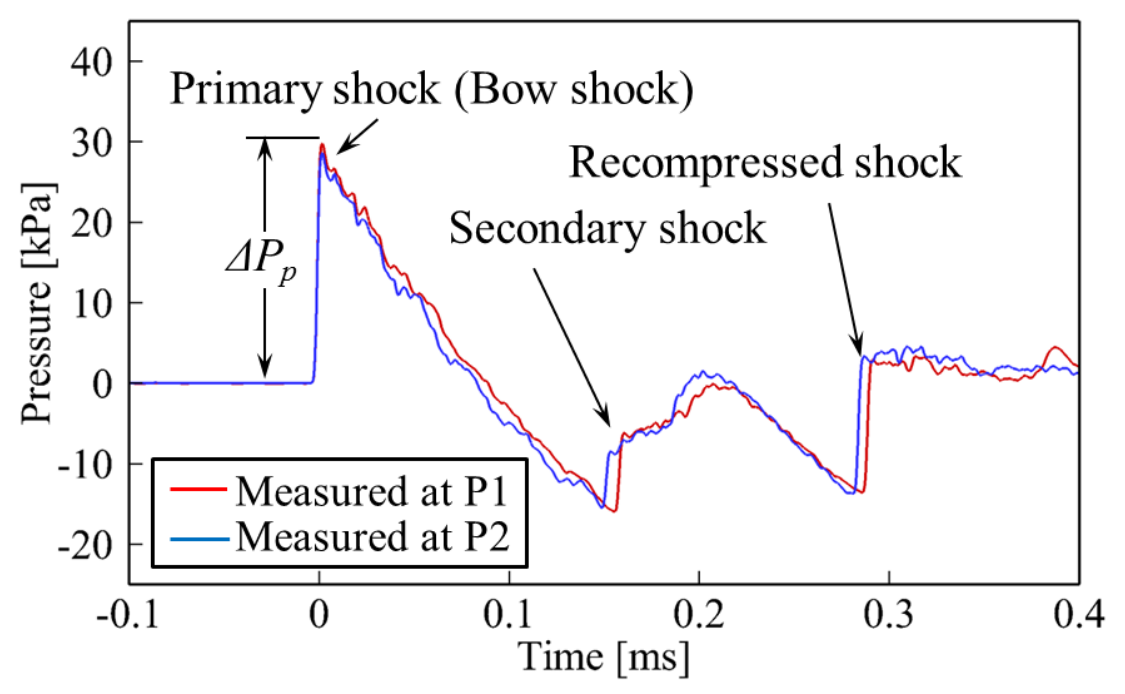

(a) Case without the turbulence interaction (shot 1)

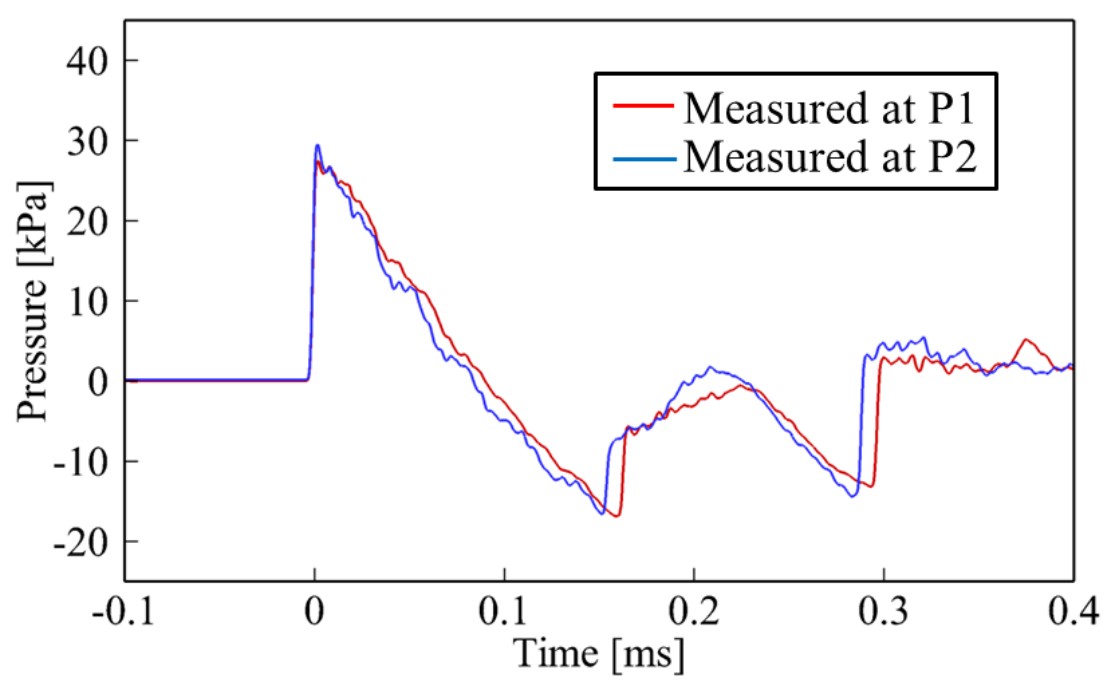

(b) Case with the turbulence interaction (shot 2)

Fig. 11 (Color online) Typical N-shaped waveforms related to the PDI images shown in Fig. 10 


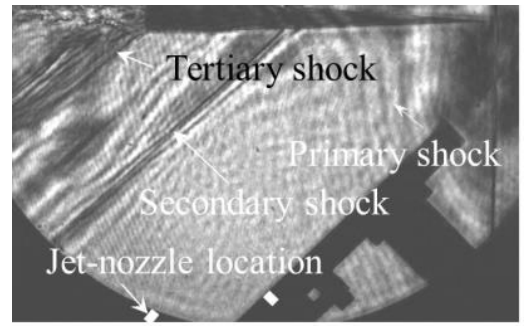

(a) $\Delta t=0 \mu \mathrm{s}$

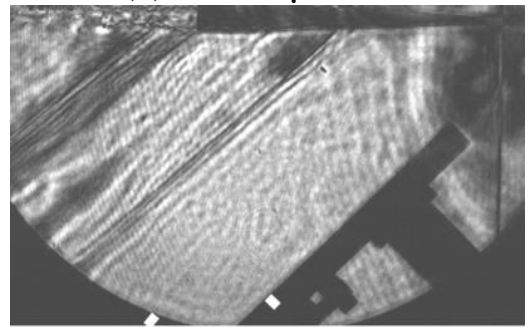

(b) $\Delta t=50 \mu \mathrm{s}$

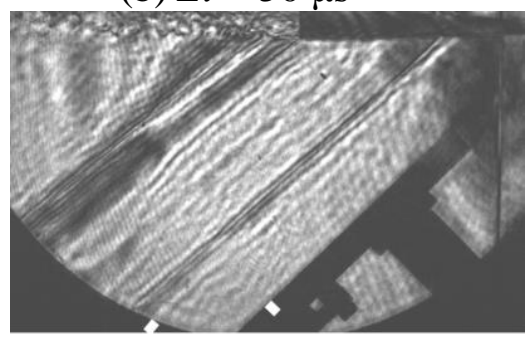

(c) $\Delta t=150 \mu \mathrm{s}$

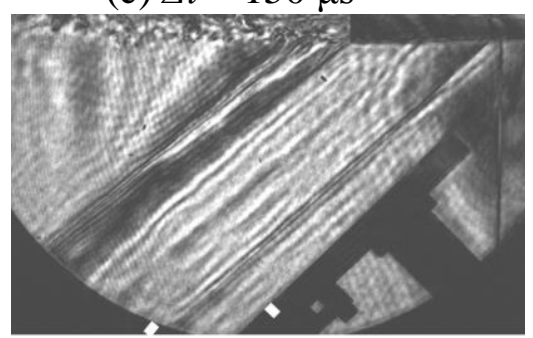

(d) $\Delta t=200 \mu \mathrm{s}$

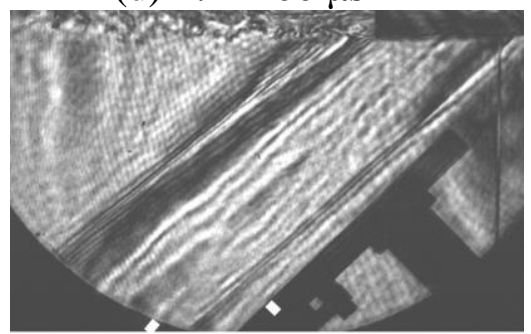

(e) $\Delta t=225 \mu \mathrm{s}$

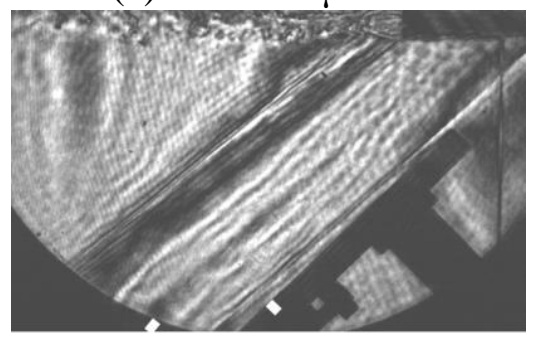

(f) $\Delta t=250 \mu \mathrm{s}$

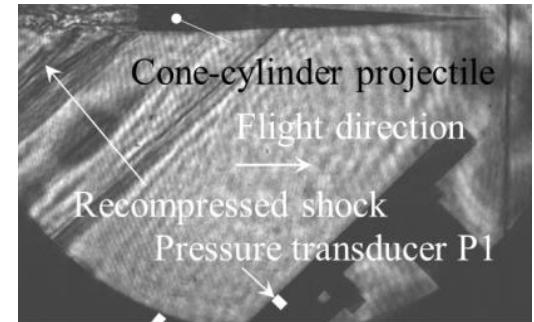

(g) $\Delta t=0 \mu \mathrm{s}$

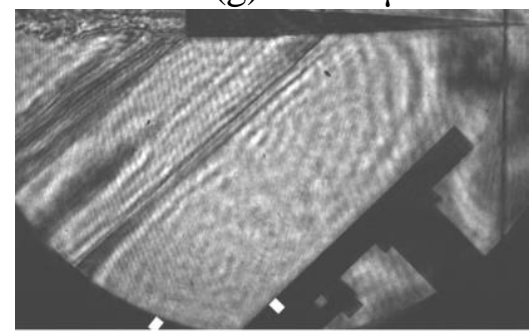

(h) $\Delta t=50 \mu \mathrm{s}$

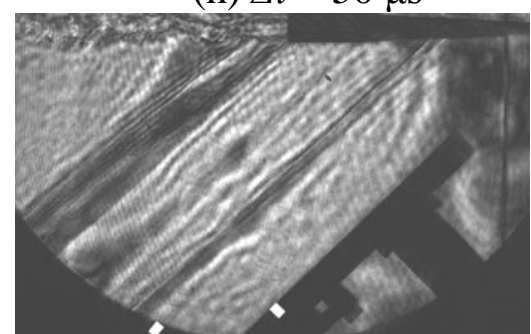

(i) $\Delta t=150 \mu \mathrm{s}$

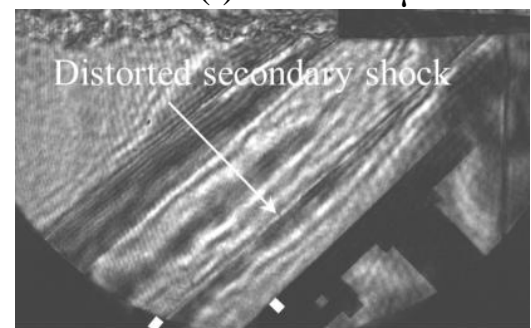

(j) $\Delta t=200 \mu \mathrm{s}$

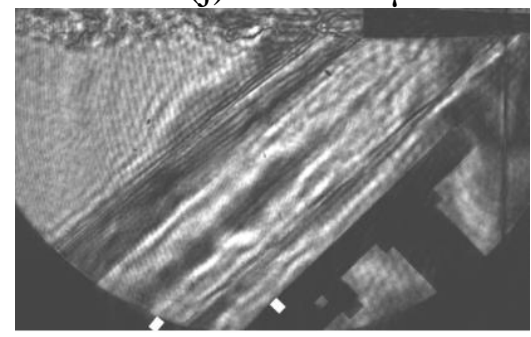

(k) $\Delta t=225 \mu \mathrm{s}$

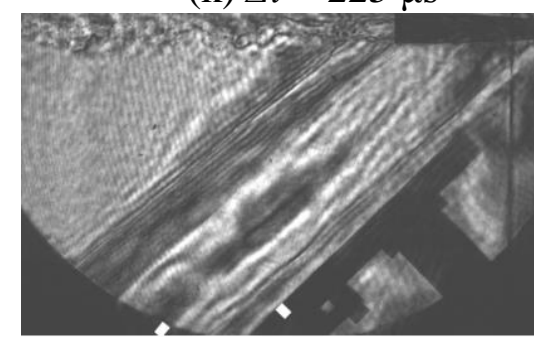

(m) $\Delta t=250 \mu \mathrm{s}$

Fig. 12 Sequential PDI visualization images around the cone-cylinder projectile; the left (a-f) and right (g-m) images correspond to the cases without (shot 3) and with (shot 4) the turbulence interaction, respectively 


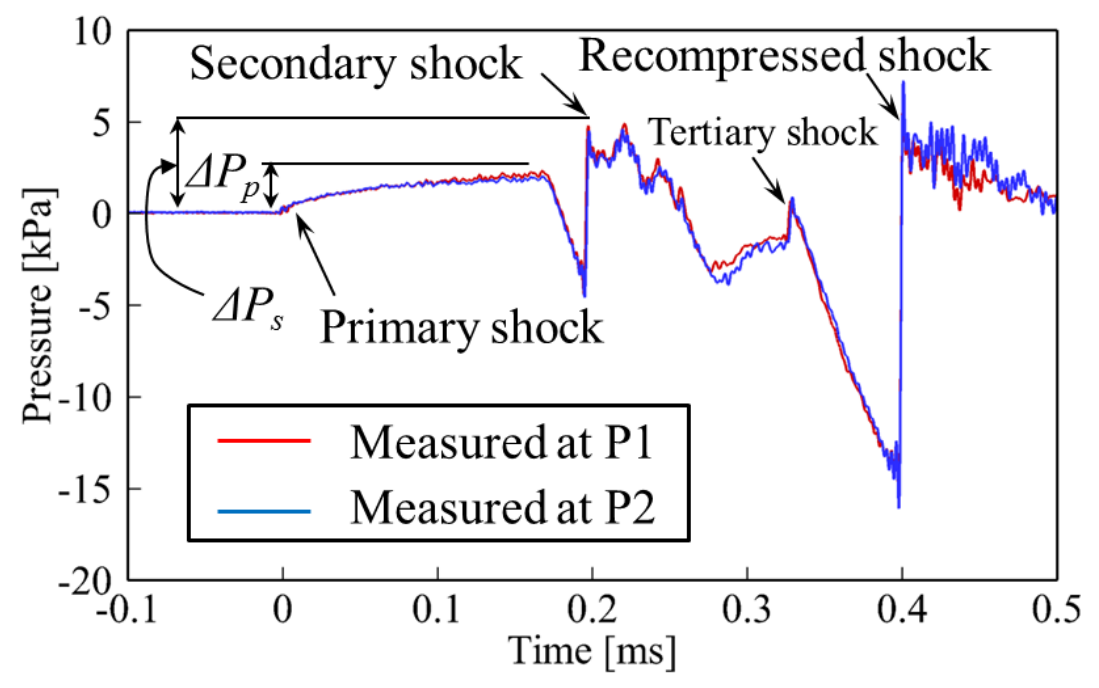

(a) Case without the turbulence interaction (shot 5)

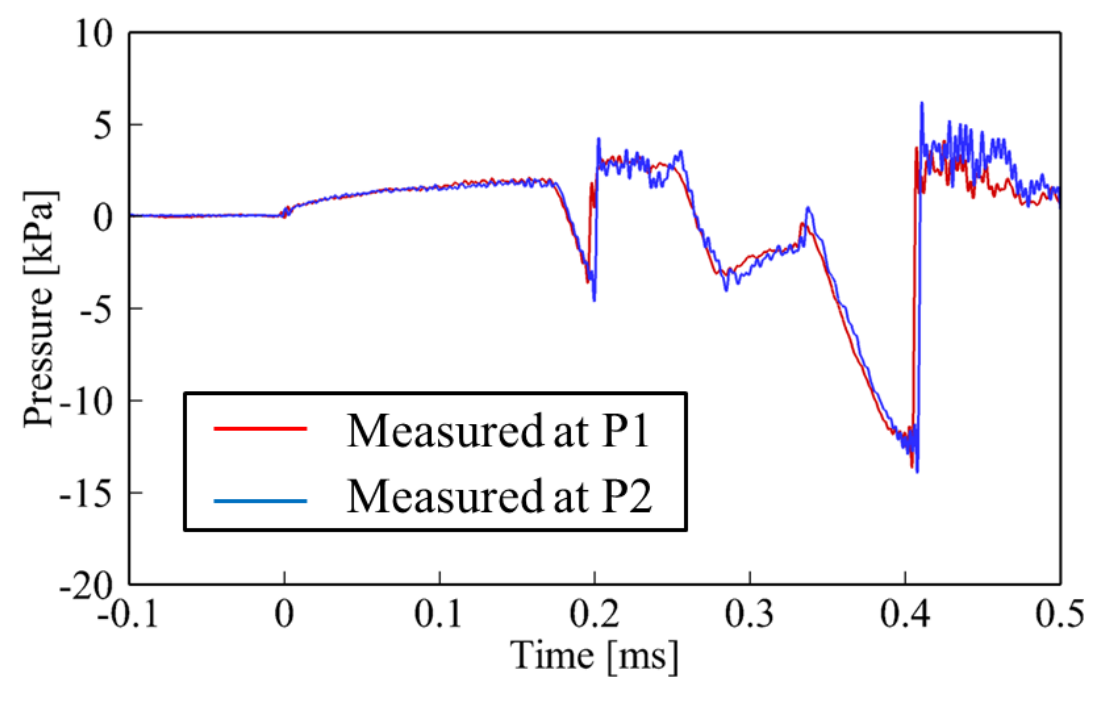

(b) Case with the turbulence interaction (shot 6)

Fig. 13 (Color online) Typical long rise-time pressure waveforms 


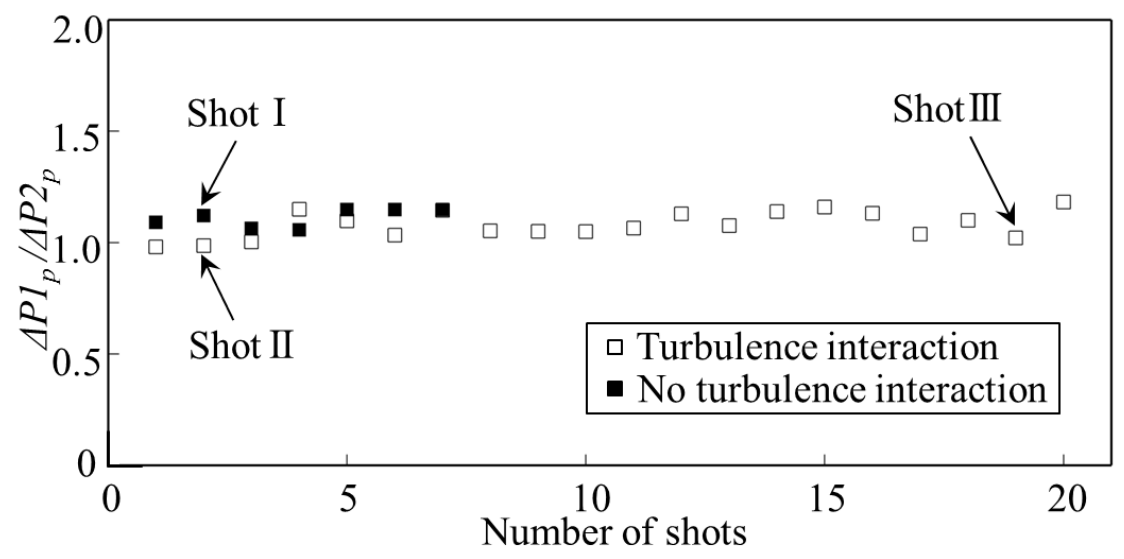

(a) Primary shock

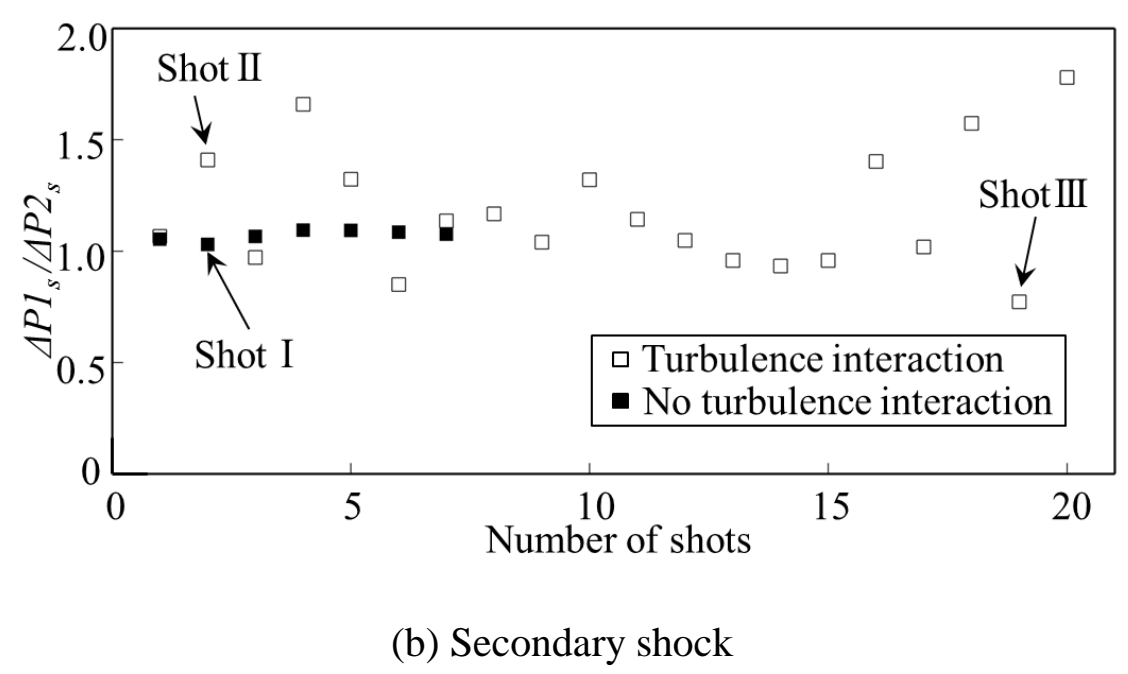

Fig. 14 Relative overpressure distributions 


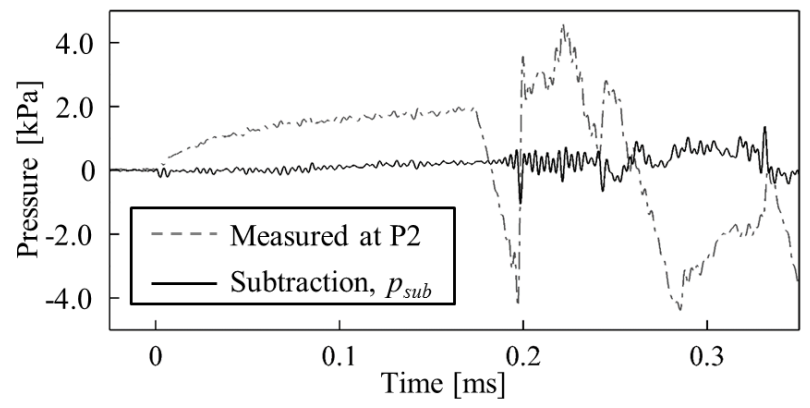

(a) Shot I , case without the turbulence interaction
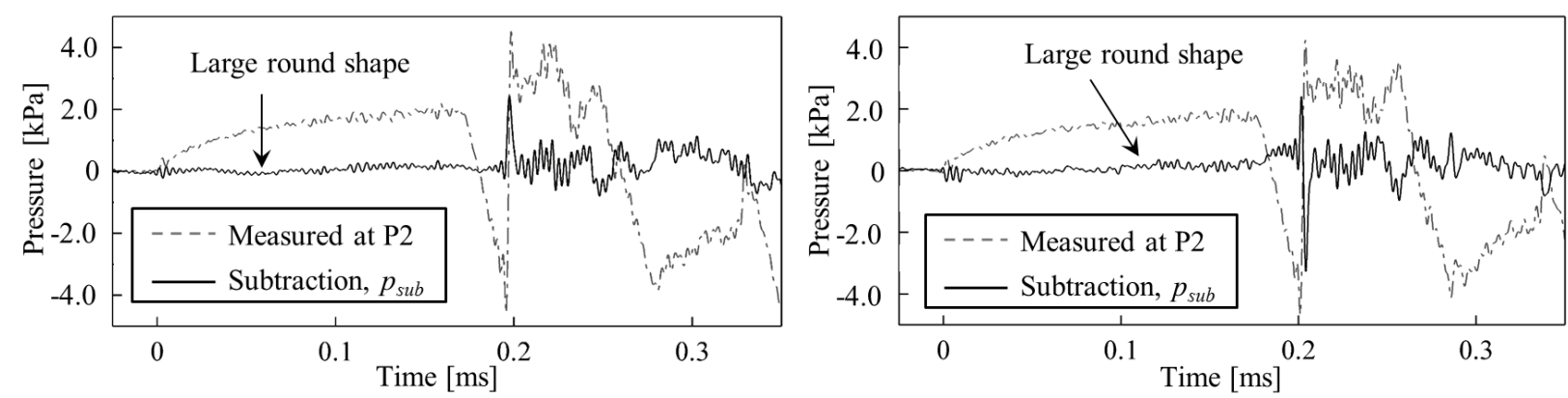

(b) Shot II , case with the turbulence interaction (c) Shot III, case with the turbulence interaction

Fig. 15 Typical subtracted pressure waveforms 


\section{Tables}

Table 1 Comparison of the spatial length scales with the sonic boom propagation in the real atmosphere

\begin{tabular}{ccc}
\hline & Real sonic boom & Present experiment \\
\cline { 2 - 3 } Shock propagation distance & $10 \mathrm{~km}$ & $200 \mathrm{~mm}$ \\
Thickness of the turbulence field & $1-2 \mathrm{~km}$ & $40 \mathrm{~mm}$ \\
Outer length scale & $100-200 \mathrm{~m}[43,44]$ & $4.2 \mathrm{~mm}$ \\
Inner length scale & $100-200 \mathrm{~mm} \mathrm{[45]}$ & $20 \mu \mathrm{m}$ \\
\hline \hline
\end{tabular}

Table 2 Statistical results for the relative overpressure

\begin{tabular}{lccc}
\hline \hline & & No-turbulence interaction & Turbulence interaction \\
\cline { 3 - 4 }$\Delta P 1_{p} / \Delta P 2_{p}$ & Mean & 1.11 & 1.08 \\
& Standard deviation & 0.04 & 0.06 \\
\cline { 2 - 4 }$\Delta P 1_{s} / \Delta P 2_{s}$ & Mean & 1.07 & 1.18 \\
& Standard deviation & 0.02 & 0.28 \\
\hline \hline
\end{tabular}

